\title{
PHYSICAL AND NUMERICAL VISCOSITY FOR QUANTUM HYDRODYNAMICS*
}

\author{
ANSGAR JÜNGEL ${ }^{\dagger}$ AND JOSIPA PINA MILIŠIĆ $\ddagger$
}

\begin{abstract}
Viscous stabilizations of the quantum hydrodynamic equations are studied. The quantum hydrodynamic model consists of the conservation laws for the particle density, momentum, and energy density, including quantum corrections from the Bohm potential. Two different stabilizations are analyzed. First, viscous terms are derived using a Fokker-Planck collision operator in the Wigner equation. The existence of solutions (with strictly positive particle density) to the isothermal, stationary, one-dimensional viscous model for general data and nonhomogeneous boundary conditions is shown. The estimates depend on the viscosity and do not allow to perform the inviscid limit. Second, the numerical viscosity of the second upwind finite-difference discretization of the inviscid quantum hydrodynamic model is computed. Finally, numerical simulations using the non-isothermal, stationary, one-dimensional model of a resonant tunnelling diode show the influence of the viscosity on the solution.
\end{abstract}

Key words. quantum hydrodynamics, viscous quantum hydrodynamics, existence of stationary solutions, numerical dispersion, numerical viscosity, resonant tunnelling diode, semiconductors

AMS subject classifications. 76Y05, 35J40, 65N05

\section{Introduction}

The fluiddynamical formulation of the Schrödinger equation, which has been known since the early years of quantum mechanics [32], has been used in many applications for quantum plasmas [34] or quantum semiconductor devices [12, 14]. Quantum effects appear through terms involving the Bohm potential and containing third-order derivatives which express the dispersive structure of the equations. These terms are mathematically difficult to handle, and in fact there are only partial analytical and numerical results. This work is devoted to an analytical and numerical study of these equations when they contain additional viscosity terms originating either from particle collisions or from an upwind numerical discretization of the equations.

More specifically, we consider the scaled stationary quantum hydrodynamic (QHD) equations for the particle density $n$, the current density $J$, and the energy density ne,

$$
\begin{aligned}
\operatorname{div} J & =F_{1}, \\
\operatorname{div}\left(\frac{J \otimes J}{n}\right)+\operatorname{div} P-n \nabla V & =F_{2}, \\
\operatorname{div}\left(\frac{J}{n} \cdot(P+n e I)\right)-J \cdot \nabla V & =F_{3}, \quad x \in \Omega, t>0,
\end{aligned}
$$

where $\Omega \subset \mathbb{R}^{3}$ is a bounded domain, $I$ is the identity matrix, the (scaled) stress tensor $P$ and energy density $n e$ are given by

$$
P=n T I-\frac{\varepsilon^{2}}{12} n(\nabla \otimes \nabla) \log n, \quad n e=\frac{|J|^{2}}{2 n}+\frac{3}{2} n T-\frac{\varepsilon^{2}}{24} n \Delta \log n,
$$

*Received: January 16, 2007; accepted (in revised version): April 18, 2007. Communicated by Pierre Degond.

${ }^{\dagger}$ Institute for Analysis and Scientific Computing, Vienna University of Technology, Wiedner Hauptstr. 8-10, 1040 Wien, Austria (juengel@anum.tuwien.ac.at).

¥Institut für Mathematik, Universität Mainz, Staudingerweg 9, 55099 Mainz, Germany (milisic@ mathematik.uni-mainz.de). 
with the scaled Planck constant $\varepsilon$ and the particle temperature $T$, and $F_{1}, F_{2}$, and $F_{3}$ are some given source terms which will be made explicit below. The electric potential $V$ is self-consistently coupled to the Poisson equation

$$
\lambda^{2} \Delta V=n-C(x),
$$

where $\lambda$ is the scaled Debye length and $C(x)$ the concentration of fixed charged background ions (doping profile). Equations (1.1)-(1.4) are to be supplemented with boundary conditions for $n, J, n e$, and $V$.

The model (1.1)-(1.3) can be derived from a mixed-state Schrödinger system $[18,19]$ or from the moment equations of the Wigner-Boltzmann model with a shiftedMaxwellian closure [14]. The mixed-state Schrödinger system does not include scattering terms, so $F_{1}=F_{2}=F_{3}=0$ in the above equations. When a Caldeira-Leggett collision operator with the relaxation-time $\tau$ is employed in the Wigner equation, we obtain $[26]$

$$
F_{1}=0, \quad F_{2}=-\frac{J}{\tau}, \quad F_{3}=-\frac{2}{\tau}\left(n e-\frac{3}{2} n T_{0}\right),
$$

where $T_{0}=1$ is the scaled lattice temperature.

The QHD model is a quantum regularization of the classical Euler equations which are recovered in the semiclassical limit $\varepsilon \rightarrow 0$. This regularization is mathematically only partially understood. More precisely, the existence of solutions, both for the transient and the stationary model, has been proven only in particular situations, either for small times [25] or for initial or boundary data sufficiently close to the thermal equilibrium state $[23,24,31]$. The main mathematical difficulty, besides the highly nonlinear structure of the third-order quantum terms, is the proof of positivity (or non-negativity) of the particle density and temperature. In the stationary case, the energy estimate only provides a bound for the energy production $\int|J|^{2} /(\tau n) d x$ which does not exclude zeros of the particle density. For special boundary conditions in the one-dimensional case, even a non-existence result was shown [13].

The mathematical difficulties can also be seen in the numerical approximation of the equations. There is some numerical evidence that the equations, discretized by finite differences or finite elements, need some kind of stabilization. In view of the similarity of the (transient) quantum hydrodynamic model to the Euler equations, the use of a hyperbolic scheme, treating the third-order quantum part as a perturbation, seems to be appropriate. For instance, Gardner [14] employed the second upwind scheme to the stationary one-dimensional quantum model, which introduces numerical viscosity. We will show that the numerical viscosity is (in one space dimension) of the form $h\left(|u| n_{x}\right)_{x} / 2$ in (1.1) and $h\left(|u|(n u)_{x}\right)_{x}$ in (1.2), where $h$ is the mesh size and $u=J / n$ is the velocity (see section 4.2 ).

Instead of employing a numerical viscosity stabilization we study in this paper the stabilization by a physical viscosity. Employing a Fokker-Planck-type collision operator [6] and Gardner's shifted-Maxwellian closure in the corresponding Wigner model, we show in section 2 that the source terms become

$$
\begin{aligned}
& F_{1}=\nu \Delta n, \quad F_{2}=-\frac{J}{\tau}+\nu \Delta J-\mu \nabla n, \\
& F_{3}=-\frac{2}{\tau}\left(n e-\frac{3}{2} n T_{0}\right)+\nu \Delta(n e)-\mu \operatorname{div} J,
\end{aligned}
$$

where $\nu>0$ is the (scaled) viscosity constant and $\mu$ is proportional to $\nu / \varepsilon$ (see section 2 for details on the derivation and the scaling of the equations). Notice that the 
viscous terms in $F_{1}$ and $F_{2}$ are similar to the numerical viscosity with $\nu$ replaced by $h|u|$. Now the effective current density is given by $J_{0}=J+\nu \nabla n$ such that $J_{0}$ is divergence-free. Equations (1.1)-(1.4) with the above expressions for $F_{1}, F_{2}$, and $F_{3}$ are called the viscous quantum hydrodynamic (QHD) equations.

The use of physical viscosity has some important consequences. First, we are able to show the existence of steady-state solutions to the one-dimensional isothermal model with physically motivated boundary conditions for any value of $\nu>0$ and any value of the applied voltage or $J_{0}$. This result is new in the literature for two reasons. First, existence of solutions to the viscous QHD equations was up to now only proved for sufficiently small $\left|J_{0}\right|[20]$. Second, we do not use no-flux boundary conditions, which simplify the analysis considerably, but we employ physically motivated boundary conditions. Namely, we assume that the particle density $n$ is given at the boundary, that $n$ satisfies as in [14] homogeneous Neumann boundary conditions, and that the second derivative $n_{x x}$ is periodic at the boundary. Furthermore, the electric potential on the boundary is given by the applied voltage. Note that there are not many mathematical results on nonlinear third-order boundary-value problems.

The main difficulties which we needed to overcome are (i) the third-order differential operator, (ii) the strong nonlinearities, and (iii) the nonhomogeneous boundary conditions. The first idea of the proof-of-existence result is to formulate the QHD equations as in [22] as a fourth-order differential equation for the exponential variable $n=e^{w}$. This avoids the difficulty (i). The fixed-point operator in the existence proof is then defined in the variable $w=\ln n$ with domain $H_{\mathrm{per}}^{2}$, the space of periodic $H^{2}$ functions. The second idea, which allows to treat the difficulty (ii), consists in estimating the energy production

$$
P=\nu \int_{0}^{1}\left(n u_{x}^{2}+\frac{\varepsilon^{2}}{3}(\sqrt{n})_{x x}^{2}+\frac{\varepsilon^{2}}{144} \frac{n_{x}^{4}}{n^{3}}+\frac{n^{2}}{2 \nu \lambda^{2}}+4\left(T+\frac{\nu}{\tau}\right)(\sqrt{n})_{x}^{2}+\frac{J_{0}^{2}}{\nu \tau n}\right) d x,
$$

where $J_{0}=J+\nu n_{x}$, using the third-order formulation of the viscous QHD model. Because of the boundary conditions (difficulty (iii)), we obtain in the first instance only the estimate $P \leq J_{0} U / \nu+c$, where $c>0$ is a constant. However, the electric power $J_{0} U / \nu$ can be estimated, thanks to the Poisson equation, in terms of the integral $\int n^{2} d x$ which can be absorbed by the energy production.

The bound on the energy production only provides an estimate for $\sqrt{n}$ in $H^{2}$; however, the definition of the fixed-point operator makes necessary an estimate for $w=\ln n$ in $H^{2}$. This is achieved by first estimating the velocity $J_{0} / n$, employing the energy production bound, and then carefully proving a bound for $w_{x x}$ in $L^{2}$, by using the fourth-order formulation of the equation. These estimates are sufficient to apply the Leray-Schauder fixed-point theorem.

We notice that the proof-of-existence result makes use of both the original thirdorder and the fourth-order formulation of the QHD model. Furthermore, we remark that all a priori bounds depend on $\nu$ and become useless in the limit $\nu \rightarrow 0$. The above energy estimate shows the regularizing effect of the viscosity since we obtain (viscosity-dependent) a priori $H^{2}$ bounds. The analysis of viscous regularizations in related third-order Korteweg models has been studied in [4]; however, their estimates cannot be used here. Our proof seems to be only valid in the one-dimensional setting since we use the embedding $H^{1} \hookrightarrow L^{\infty}$. Moreover, it seems to be difficult to generalize the proof to the viscous QHD model including the energy equation (1.3).

The second consequence of the viscous terms concerns the numerical approximation of the QHD model. The inviscid equations have been discretized employing 
an upwind finite-difference approximation [14], a Runge-Kutta discontinuous Galerkin method [7, 8], and a relaxation scheme [28]. However, we found that the QHD model is very sensitive with respect to a change of the physical viscosity. As mentioned above, these hyperbolic-type schemes lead to possibly large numerical viscosity. Therefore, we employ a simple central finite-difference scheme which avoids numerical viscosity. We show in section 4.2 that the numerical dispersion, introduced by the central scheme, is much smaller than the physical dispersion, given by the third-order quantum terms.

We are aware that a central finite-difference approximation is a rather simple numerical method. However, it provides good numerical results and we give, for the first time, a consistency analysis which allows to interpret the numerical results for both the central and the upwind discretization. More advanced numerical methods are either a steady-state Newton solver using ENO or WENO, or the Runge-Kutta discontinuous Galerkin method [17].

The analytical results do not allow for the inviscid limit $\nu \rightarrow 0$. Numerically this can be seen by the fact that the viscosity cannot be chosen arbitrarily small in our scheme (although the physical value for $\nu$ can be used). This indicates that the physical viscosity is indeed needed for stabilization.

There is also a drawback of the viscous model. One of the interests of the QHD equations is the simulation of resonant tunnelling diodes whose current-voltage characteristics exhibit so-called negative differential resistance effects, i.e., the current density is monotonically decreasing in a certain voltage range. An important quantity is the peak-to-valley ratio, i.e. the quotient of local maximal to local minimal current densities. Our numerical experiments for the non-isothermal model show that the peak-to-valley ratio is too small compared to physical experiments [30]. Thus, the physical viscosity strongly influences the quantum behavior of the model. We notice that more physical current-voltage characteristics can be obtained by the "smooth" QHD model of Gardner and Ringhofer [15, 16].

The paper is organized as follows. In section 2 we sketch the derivation of the viscous QHD equations and detail the scaling. Section 3 is devoted to the proof of the existence of solutions to the isothermal model in one space dimension. In section 4 some numerical results for the isothermal and the non-isothermal model are presented. More precisely, finite-difference approximations of the viscous and inviscid model are proposed, and the numerical viscosity and the numerical dispersion of the schemes are computed. Moreover, numerical simulations of a resonant tunnelling diode are presented.

\section{Modeling-scaling}

The viscous QHD equations are derived from the Wigner-Fokker-Planck equation for the distribution function $f(x, p, t)$,

$$
\partial_{t} f+\frac{p}{m} \cdot \nabla_{x} f+\frac{q}{m} \theta[V] f=Q_{F P}(f), \quad(x, p) \in \mathbb{R}^{6}, t>0,
$$

where $(x, p)$ denotes the position-momentum variables, $t>0$ the time, and $\theta[V]$ is a pseudo-differential operator defined by

$$
\begin{aligned}
& (\theta[V] f)(x, p, t) \\
= & i \\
(2 \pi)^{3 / 2} & \int_{\mathbb{R}^{6}} \frac{m}{\hbar}\left[V\left(x+\frac{\hbar}{2} \eta, t\right)-V\left(x-\frac{\hbar}{2} \eta, t\right)\right] f\left(x, p^{\prime}, t\right) \mathrm{e}^{i \eta \cdot\left(p-p^{\prime}\right)} d \eta d p^{\prime}
\end{aligned}
$$

(see [20]). The physical constants are the effective electron mass $m$, the elementary charge $q$, and the reduced Planck constant $\hbar$. The function $V(x, t)$ is the electric 
potential, selfconsistently given by the Poisson equation

$$
\varepsilon_{s} \Delta_{x} V=q\left(\hbar^{-3} \int_{\mathbb{R}^{3}} f d p-C(x)\right)
$$

where $\varepsilon_{s}$ denotes the permittivity of the semiconductor material and $C(x)$ is the doping profile. We assume that the collision operator $Q_{F P}(f)$ models the interaction of the electrons with the phonons of the crystal lattice (oscillators) and that it is given by the Fokker-Planck expression [6]

$$
Q_{F P}(f)=\frac{1}{\tau_{0}} \operatorname{div}_{p}(p f)+D_{p p} \Delta_{p} f+2 D_{p q} \operatorname{div}_{x}\left(\nabla_{p} f\right)+D_{q q} \Delta_{x} f,
$$

where $\tau_{0}$ is a friction parameter (relaxation time) and the constants

$$
D_{p p}=\frac{m k_{B} T_{0}}{\tau_{0}}, \quad D_{p q}=\frac{\Omega \hbar^{2}}{12 \pi k_{B} T_{0} \tau_{0}}, \quad D_{q q}=\frac{\hbar^{2}}{12 m k_{B} T_{0} \tau_{0}}
$$

constitute the phase-space diffusion matrix. Here, $T_{0}$ is the lattice temperature and $\Omega$ the cut-off frequency of the reservoir oscillators. If $D_{p q}=0$ and $D_{q q}=0$, this gives the Caldeira-Leggett operator [5]. The above scattering operator has been derived under the assumptions that the thermal energy $k_{B} T_{0}$ is of the same order as the wave energy $\Omega \hbar$ corresponding to the cut-off frequency, and that the reservoir memory time $1 / \Omega$ is much smaller than the characteristic time scale $t^{*}$ of the electrons and the relaxation time $\tau_{0}$. For a discussion of the Wigner-Fokker-Planck model (2.1), we refer to [2].

In order to derive macroscopic equations we apply the moment method as in [14]. The idea is to multiply $(2.1)$ by $1, p$, and $\frac{1}{2}|p|^{2}$ and to integrate over $\mathbb{R}^{3}$ with respect to $p / \hbar^{3}$. The resulting system of equations is closed by Gardner's shifted quantum Maxwellian [14], which is an approximation of the quantum equilibrium state according to Wigner [35, formula (25)]. Gardner's shifted Maxwellian is a quantum mechanical analogue of the classical Maxwellian which lies in the kernel of the FokkerPlanck operator. A different quantum Maxwellian derived from quantum entropy maximization has been recently suggested by [9] (also see [26]). However, the resulting model equations are more complicated such that we prefer here the simpler choice of [14].

The only difference to the derivation in [14] is the integration of the Fokker-Planck term. This yields

$$
\begin{aligned}
\left\langle Q_{F P}(f)\right\rangle & =D_{q q} \Delta_{x}\langle f\rangle \\
\left\langle p Q_{F P}(f)\right\rangle & =-\frac{1}{\tau_{0}}\langle p f\rangle-2 D_{p q} \nabla_{x}\langle f\rangle+D_{q q} \Delta_{x}\langle p f\rangle \\
\left\langle\frac{1}{2}|p|^{2} Q_{F P}(f)\right\rangle & =-\frac{2}{\tau_{0}}\left\langle\frac{1}{2}|p|^{2} f\right\rangle+3 D_{p p}\langle f\rangle-2 D_{p q} \operatorname{div}_{x}\langle p f\rangle+D_{q q} \Delta_{x}\left\langle\frac{1}{2}|p|^{2} f\right\rangle
\end{aligned}
$$

where we used the notation

$$
\langle g(p)\rangle=\hbar^{-3} \int_{\mathbb{R}^{3}} g(p) d p
$$

for functions $g(p)$. Introducing the electron density $n=\langle f\rangle$, the electron current density $J=-(q / m)\langle p f\rangle$, and the energy density $n e=\left\langle|p|^{2}\right\rangle / 2 m$, we obtain finally the 
viscous QHD equations

$$
\begin{aligned}
\partial_{t} n-\frac{1}{q} \operatorname{div} J= & D_{q q} \Delta n, \\
& \partial_{t} J-\frac{1}{q} \operatorname{div}\left(\frac{J \otimes J}{n}\right)-\frac{q k_{B}}{m} \nabla(n T)+\frac{q^{2}}{m} n \nabla V, \\
+\frac{q \hbar^{2}}{12 m^{2}} \operatorname{div}(n(\nabla \otimes \nabla) \log n)= & -\frac{J}{\tau_{0}}+\frac{2 q D_{p q}}{m} \nabla n+D_{q q} \Delta J, \\
& \partial_{t}(n e)-\frac{1}{q} \operatorname{div}\left(\frac{J}{n}(n e+P)\right)+J \cdot \nabla V, \\
= & -\frac{2}{\tau_{0}}\left(n e-\frac{3}{2} n k_{B} T_{0}\right)+\frac{2 D_{p q}}{q} \operatorname{div} J+D_{q q} \Delta(n e), \\
\varepsilon_{s}^{2} \Delta V= & q(n-C(x)), \quad x \in \mathbb{R}^{3}, t>0,
\end{aligned}
$$

where $J \otimes J$ denotes the matrix with components $J_{j} J_{k}$, and the stress tensor and energy density are given by, respectively,

$$
\begin{aligned}
P & =n k_{B} T I-\frac{\hbar^{2}}{12 m} n(\nabla \otimes \nabla) \log n, \\
n e & =\frac{m}{2 q^{2}} \frac{|J|^{2}}{n}+\frac{3}{2} n k_{B} T-\frac{\hbar^{2}}{24 m} n \Delta \log n .
\end{aligned}
$$

The stress tensor consists of the classical pressure and a quantum "pressure" term. The energy density is the sum of kinetic energy, thermal energy, and quantum energy.

Next, we scale the above system of equations. Let $L$ be a characteristic length, for instance the device length. We define the characteristic density, voltage, and current density, respectively, by

$$
C^{*}=\sup |C|, \quad V^{*}=\frac{k_{B} T_{0}}{q}, \quad J^{*}=\frac{q k_{B} T_{0} C^{*} t^{*}}{m L},
$$

where the characteristic time $t^{*}$ is given by $k_{B} T_{0}=m L^{2} /\left(t^{*}\right)^{2}$, i.e., we assume that the thermal energy of a particle is equal to the kinetic energy needed to cross the device in time $t^{*}$. According to the conditions under which the Fokker-Planck term has been derived, we assume that the cut-off wave energy equals the thermal energy, i.e., $\Omega \hbar=k_{B} T_{0}$. Then, after introducing the scaling (notice the change of sign for the current density)

$$
x \rightarrow L x, \quad t \rightarrow t^{*} t, \quad C \rightarrow C^{*} C, \quad V \rightarrow V^{*} V, \quad J \rightarrow-J^{*} J, \quad T \rightarrow T_{0} T,
$$

we obtain the scaled equations

$$
\begin{aligned}
\partial_{t} n+\operatorname{div} J= & \nu \Delta n, \\
& \partial_{t} J+\operatorname{div}\left(\frac{J \otimes J}{n}\right)+\nabla(n T)-n \nabla V-\frac{\varepsilon^{2}}{6} n \nabla\left(\frac{\Delta \sqrt{n}}{\sqrt{n}}\right), \\
= & -\frac{J}{\tau}+\nu \Delta J-\mu \nabla n, \\
& \partial_{t}(n e)+\operatorname{div}\left(\frac{J}{n}(n e+P)\right)-J \cdot \nabla V, \\
= & -\frac{2}{\tau} n\left(e-\frac{3}{2}\right)+\nu \Delta(n e)-\mu \operatorname{div} J, \\
\lambda^{2} \Delta V= & n-C(x),
\end{aligned}
$$


where the scaled parameters are the viscosity constant $\nu$, the Planck constant $\varepsilon$, the Debye length $\lambda$, given by

$$
\nu=\frac{\hbar}{\sqrt{k_{B} T_{0} m L^{2}}} \frac{\hbar}{12 k_{B} T_{0} \tau_{0}}, \quad \varepsilon^{2}=\frac{\hbar^{2}}{k_{B} T_{0} m L^{2}}, \quad \lambda^{2}=\frac{\varepsilon_{s} k_{B} T_{0}}{q^{2} C^{*} L^{2}},
$$

and the relaxation time and interaction constant, respectively,

$$
\tau=\frac{\tau_{0}}{t^{*}}, \quad \mu=\frac{\hbar}{6 \pi k_{B} T_{0} \tau_{0}} .
$$

The scaled stress tensor and energy density can be expressed as

$$
P=n T I-\frac{\varepsilon^{2}}{12} n(\nabla \otimes \nabla) \log n, \quad n e=\frac{|J|^{2}}{2 n}+\frac{3}{2} n T-\frac{\varepsilon^{2}}{24} n \Delta \log n .
$$

In the following sections we will study the one-dimensional stationary equations in a bounded domain.

\section{Analytical results}

In this section we consider the stationary viscous QHD equations with constant temperature $T$ in one space dimension,

$$
\begin{aligned}
J_{x} & =\nu n_{x x}, \\
\left(\frac{J^{2}}{n}\right)_{x}+T n_{x}-n V_{x}-\frac{\varepsilon^{2}}{6} n\left(\frac{(\sqrt{n})_{x x}}{\sqrt{n}}\right)_{x} & =-\frac{J}{\tau}+\nu J_{x x}, \\
\lambda^{2} V_{x x} & =n-C(x) \quad \text { in }(0,1),
\end{aligned}
$$

with the physically motivated boundary conditions

$$
\begin{aligned}
& n(0)=n(1)=1, \quad n_{x}(0)=n_{x}(1), \quad n_{x x}(0)=n_{x x}(1), \\
& V(0)=0, \quad V(1)=U .
\end{aligned}
$$

Notice that the term $\mu n_{x}$ in (2.7) can be absorbed by $T n_{x}$. The first two boundary conditions express that the total space charge vanishes at the boundary (if $C(0)=$ $C(1)=1$ ). The remaining conditions on $n$ can be justified if the doping profile is nearly constant close to the boundary points (see section 4.3). Notice that (3.1) and the boundary condition $n_{x}(0)=n_{x}(1)$ imply that the current density takes the same value at $x=0$ and $x=1$, namely $J(0)=J(1)=J_{0}$ for some constant $J_{0}$. Given the applied voltage $U$, the effective current density $J_{0}$ can be computed from the solution of the above boundary-value problem, which gives a well-defined currentvoltage characteristic.

It is not difficult to see that the number of boundary conditions for the system (3.1)-(3.3) is correct. Indeed, (3.1) implies that $J=\nu n_{x}+J_{0}$, which gives

$$
\left(\frac{J^{2}}{n}\right)_{x}-\nu J_{x x}=-2 \nu^{2} n\left(\frac{(\sqrt{n})_{x x}}{\sqrt{n}}\right)_{x}+\left(\frac{J_{0}^{2}}{n}\right)_{x}+2 \nu J_{0}(\log n)_{x x},
$$

and hence (3.2) can be written as

$$
\begin{aligned}
& \left(\frac{J_{0}^{2}}{n}\right)_{x}+\left(T+\frac{\nu}{\tau}\right) n_{x}-n V_{x}-\left(2 \nu^{2}+\frac{\varepsilon^{2}}{6}\right) n\left(\frac{(\sqrt{n})_{x x}}{\sqrt{n}}\right)_{x} \\
= & -\frac{J_{0}}{\tau}-2 J_{0} \nu(\log n)_{x x} .
\end{aligned}
$$


Therefore, the system (3.1)-(3.3) is (formally) equivalent to the system (3.6) and (3.3) for which five boundary conditions are sufficient.

The left-hand side of (3.6) equals the usual QHD equations except that the scaled Planck constant and the scaled temperature are larger. The viscosity $\nu$ has the effect of increasing the Bohm potential and the pressure. Moreover, the right-hand side of (3.6) contains the diffusion term $2 J_{0} \nu(\log n)_{x x}$. Thus, the viscous QHD equations can be interpreted as a quantum fluid model with larger scaled Planck constant and with a diffusion term in the variable $\log n$. We notice that QHD models with various diffusion terms in $n$ (but not in $\log n$ ) have been studied in [13].

Our main result is the following existence theorem.

Theorem 3.1. Let $C \in L^{2}(0,1)$ and let $T, \varepsilon, \tau$, and $\lambda$ be positive constants. Then there exists for any $U \in \mathbb{R}$ and any $\nu>0$ a solution $(n, J, V) \in H^{4}(0,1) \times H^{3}(0,1) \times$ $H^{2}(0,1)$ to (3.1)-(3.5) satisfying $n>0$ in $(0,1)$. Moreover,

$$
\|\sqrt{n}\|_{H^{2}}+\|\log n\|_{H^{2}}+\left\|\frac{J_{0}}{n}\right\|_{L^{\infty}} \leq c \nu^{-1}
$$

where $J_{0}=J-\nu n_{x} \in \mathbb{R}$ and $c>0$ is a constant depending only on the data but not on $\nu$.

3.1. Reformulation of the equations. The first idea for the proof, taken from [22], is to formulate the system (3.6) and (3.3) as a single fourth-order equation, in which the Poisson equation is taken into account. We divide (3.6) by $n$, differentiate the resulting equation with respect to $x$ and employ the Poisson equation (3.3):

$$
\begin{aligned}
& -\left(2 \nu^{2}+\frac{\varepsilon^{2}}{6}\right)\left(\frac{(\sqrt{n})_{x x}}{\sqrt{n}}\right)_{x x}+\left(T+\frac{\nu}{\tau}\right)(\log n)_{x x} \\
= & \frac{n-C}{\lambda^{2}}+J_{0}^{2}\left(\frac{n_{x}}{n^{3}}\right)_{x}-2 J_{0} \nu\left(\frac{1}{n}(\log n)_{x x}\right)_{x}-\frac{J_{0}}{\tau}\left(\frac{1}{n}\right)_{x} .
\end{aligned}
$$

The electric potential can be recovered from (3.6), after division by $n$ and integration, taking into account the boundary conditions $n(0)=1$ and $V(0)=0$,

$$
\begin{aligned}
V(x)= & -\left(2 \nu^{2}+\frac{\varepsilon^{2}}{6}\right) \frac{(\sqrt{n})_{x x}(x)}{\sqrt{n}(x)}+\left(T+\frac{\nu}{\tau}\right) \log n(x)+\frac{J_{0}^{2}}{2 n(x)^{2}} \\
& +\frac{J_{0}}{\tau} \int_{0}^{x} \frac{d s}{n}+2 J_{0} \nu \frac{n_{x}(x)}{n(x)^{2}}+2 J_{0} \nu \int_{0}^{x} \frac{n_{x}^{2}}{n^{3}} d s \\
& +\left(2 \nu^{2}+\frac{\varepsilon^{2}}{6}\right)(\sqrt{n})_{x x}(0)-\frac{J_{0}^{2}}{2}-2 J_{0} \nu n_{x}(0) .
\end{aligned}
$$

Introducing the new variable $n=\mathrm{e}^{w}$ and observing that

$$
\left(\frac{(\sqrt{n})_{x x}}{\sqrt{n}}\right)_{x x}=\frac{1}{2}\left(\frac{1}{n}\left(n(\log n)_{x x}\right)_{x}\right)_{x}=\frac{1}{2}\left(w_{x x}+\frac{w_{x}^{2}}{2}\right)_{x x},
$$

we can write (3.7)-(3.8) as

$$
\begin{aligned}
& -\left(\nu^{2}+\frac{\varepsilon^{2}}{12}\right)\left(w_{x x}+\frac{w_{x}^{2}}{2}\right)_{x x}+\left(T+\frac{\nu}{\tau}\right) w_{x x} \\
= & \lambda^{-2}\left(\mathrm{e}^{w}-C\right)+J_{0}^{2}\left(\mathrm{e}^{-2 w} w_{x}\right)_{x}-2 J_{0} \nu\left(\mathrm{e}^{-w} w_{x x}\right)_{x}-\frac{J_{0}}{\tau}\left(\mathrm{e}^{-w}\right)_{x},
\end{aligned}
$$


and

$$
\begin{aligned}
V(x)= & -\left(\nu^{2}+\frac{\varepsilon^{2}}{12}\right)\left(w_{x x}(x)+\frac{w_{x}(x)^{2}}{2}\right)+\left(T+\frac{\nu}{\tau}\right) w(x)+\frac{J_{0}^{2}}{2} \mathrm{e}^{-2 w(x)} \\
& +\frac{J_{0}}{\tau} \int_{0}^{x} \mathrm{e}^{-w(s)} d s+2 J_{0} \nu \mathrm{e}^{-w(x)} w_{x}(x)+2 J_{0} \nu \int_{0}^{x} \mathrm{e}^{-w(s)} w_{x}(s)^{2} d s \\
& +\left(\nu^{2}+\frac{\varepsilon^{2}}{12}\right)\left(w_{x x}(0)+\frac{w_{x}(0)^{2}}{2}\right)-\frac{J_{0}^{2}}{2}-2 J_{0} \nu w_{x}(0) .
\end{aligned}
$$

Equation (3.9) has to be solved in the interval $(0,1)$ with the boundary conditions

$$
w(0)=w(1)=0, \quad w_{x}(0)=w_{x}(1), \quad w_{x x}(0)=w_{x x}(1) .
$$

We have shown the first part of the following lemma.

LEMmA 3.2. Let $(n, J, V)$ be a smooth solution to (3.1)-(3.5) such that $n>0$ in $(0,1)$. Then $w=\log n$ is a solution to (3.9) with boundary conditions (3.11), $V$ solves (3.10), and $J_{0}$ is defined by (3.8) with $x=1$, i.e.

$$
U=\frac{J_{0}}{\tau} \int_{0}^{1} \frac{d x}{n}+2 J_{0} \nu \int_{0}^{1} \frac{n_{x}^{2}}{n^{3}} d x .
$$

Conversely, if $w \in H^{4}(0,1)$ is a solution to (3.9) with boundary conditions (3.11) and if $V$ and $J_{0}$ are given by, respectively, (3.10) and (3.12), then $\left(\mathrm{e}^{w}, J, V\right) \in H^{4}(0,1) \times$ $H^{3}(0,1) \times H^{2}(0,1)$ is a solution to (3.1)-(3.5), where $J:=\nu \mathrm{e}^{w} w_{x}+J_{0}$.

Proof. It remains to show that $\left(\mathrm{e}^{w}, J, V\right)$ solves (3.1)-(3.5). In order to see this, first differentiate (3.10) twice with respect to $x$ and take the difference with (3.9). This yields the Poisson equation (3.3). Then differentiate (3.10) once with respect to $x$ and multiply the resulting equation by $n$. This yields (3.6). Defining $J=\nu n_{x}+J_{0}$, we see that (3.6) is equivalent to (3.2). Moreover, differentiating $J$ with respect to $x$ gives (3.1). The boundary conditions (3.4) are a consequence of (3.11). Finally, the boundary conditions (3.5) are obtained by taking $x=0$ and $x=1$ in (3.10) and using (3.12).

3.2. Uniform estimates. For the proof of Theorem 3.1 we need some uniform estimates. Our second idea of the existence analysis is to show that the energy production is bounded, which provides the necessary estimates.

Lemma 3.3. Let $(n, J, V) \in H^{4}(0,1) \times H^{3}(0,1) \times H^{2}(0,1)$ be a solution to $(3.1)-(3.5)$ such that $n>0$ in $(0,1)$. Then there exists a constant $c>0$ such that

$$
\begin{aligned}
\int_{0}^{1}( & \frac{\left(J_{x} n-J n_{x}\right)^{2}}{n^{3}}+\frac{\varepsilon^{2}}{3}(\sqrt{n})_{x x}^{2}+\frac{\varepsilon^{2}}{144} \frac{n_{x}^{4}}{n^{3}}+\frac{n^{2}}{4 \lambda^{2}}+4\left(T+\frac{\nu}{\tau}\right)(\sqrt{n})_{x}^{2} \\
& \left.+\frac{J_{0}^{2}}{\nu \tau n}\right) d x \leq c \nu^{-2},
\end{aligned}
$$

where $c>0$ only depends on $\tau, \lambda, U$, and $\|C\|_{L^{2}}$.

The integrand of the above inequality can indeed be interpreted as the energy production: In [21] is has been shown that, with thermal equilibrium boundary conditions, the energy of the time-dependent viscous QHD equations is bounded for all time and the energy production is given by the above integral. We notice that, setting $J=n u$, the first integrand can be written as $\int_{0}^{1} n u_{x}^{2} d x$. 
Proof. We multiply (3.1) by $T \log n-J^{2} / 2 n^{2}-V-\varepsilon^{2}(\sqrt{n})_{x x} /(6 \sqrt{n})$ and integrate by parts to obtain

$$
\begin{aligned}
& \int_{0}^{1}\left(-T J \frac{n_{x}}{n}-\frac{J^{2} J_{x}}{2 n^{2}}+J V_{x}-\frac{\varepsilon^{2}}{6} \frac{(\sqrt{n})_{x x}}{\sqrt{n}} J_{x}\right) d x+[J T \log n-J V]_{0}^{1} \\
= & \nu \int_{0}^{1}\left(-T \frac{n_{x}^{2}}{n}+\left(\frac{J^{2}}{2 n^{2}}\right)_{x} n_{x}+n_{x} V_{x}-\frac{\varepsilon^{2}}{6} \frac{(\sqrt{n})_{x x}}{\sqrt{n}} n_{x x}\right) d x \\
& +\nu\left[n_{x} T \log n-n_{x} \frac{J^{2}}{2 n^{2}}-n_{x} V\right]_{0}^{1} .
\end{aligned}
$$

Taking into account the boundary conditions (3.4)-(3.5) and $J(0)=J(1)$, the above equation becomes

$$
\begin{aligned}
& \int_{0}^{1}\left(-T J \frac{n_{x}}{n}-\frac{J^{2} J_{x}}{2 n^{2}}+J V_{x}-\frac{\varepsilon^{2}}{6} \frac{(\sqrt{n})_{x x}}{\sqrt{n}} J_{x}\right) d x-J(1) U \\
= & \nu \int_{0}^{1}\left(-T \frac{n_{x}^{2}}{n}+\left(\frac{J^{2}}{2 n^{2}}\right)_{x} n_{x}+n_{x} V_{x}-\frac{\varepsilon^{2}}{6} \frac{(\sqrt{n})_{x x}}{\sqrt{n}} n_{x x}\right) d x-\nu n_{x}(1) U .
\end{aligned}
$$

Furthermore, we multiply (3.2) by $J / n$ and integrate by parts:

$$
\begin{aligned}
& \int_{0}^{1}\left(\left(\frac{J^{2}}{n}\right)_{x} \frac{J}{n}+T J \frac{n_{x}}{n}-J V_{x}+\frac{\varepsilon^{2}}{6} \frac{(\sqrt{n})_{x x}}{\sqrt{n}} J_{x}\right) d x-\frac{\varepsilon^{2}}{6}\left[\frac{(\sqrt{n})_{x x}}{\sqrt{n}} J\right]_{0}^{1} \\
= & \int_{0}^{1}\left(-\frac{J^{2}}{\tau n}-\nu J_{x}\left(\frac{J}{n}\right)_{x}\right) d x+\left[\nu J_{x} \frac{J}{n}\right]_{0}^{1} .
\end{aligned}
$$

Taking into account the boundary conditions (3.4) and $J_{x}=\nu n_{x x}$, the boundary terms vanish. Hence, adding (3.13) and (3.14) gives, since some terms cancel,

$$
\begin{aligned}
& \int_{0}^{1}\left(-\frac{J^{2} J_{x}}{2 n^{2}}+\left(\frac{J^{2}}{n}\right)_{x} \frac{J}{n}\right) d x+\nu \int_{0}^{1}\left(J_{x}\left(\frac{J}{n}\right)_{x}-\left(\frac{J^{2}}{2 n^{2}}\right)_{x} n_{x}\right) d x \\
& +\nu \frac{\varepsilon^{2}}{6} \int_{0}^{1} \frac{(\sqrt{n})_{x x}}{\sqrt{n}} n_{x x} d x-\nu \int_{0}^{1} n_{x} V_{x} d x+\int_{0}^{1}\left(\nu T \frac{n_{x}^{2}}{n}+\frac{J^{2}}{\tau n}\right) d x \\
= & J(1) U-\nu n_{x}(1) U=J_{0} U .
\end{aligned}
$$

The first integral vanishes since

$$
\begin{aligned}
\int_{0}^{1}\left(-\frac{J^{2} J_{x}}{2 n^{2}}+\left(\frac{J^{2}}{n}\right)_{x} \frac{J}{n}\right) d x & =\frac{1}{2} \int_{0}^{1}\left(\frac{J^{3}}{n^{2}}\right)_{x} d x=\frac{1}{2}\left[\frac{J^{3}}{n^{2}}\right]_{0}^{1} \\
& =\frac{1}{2}\left(J(1)^{3}-J(0)^{3}\right)=0
\end{aligned}
$$

The second integral equals

$$
\nu \int_{0}^{1}\left(J_{x}\left(\frac{J}{n}\right)_{x}-\left(\frac{J^{2}}{2 n^{2}}\right)_{x} n_{x}\right) d x=\nu \int_{0}^{1} \frac{1}{n^{3}}\left(J_{x} n-J n_{x}\right)^{2} d x .
$$

Since, using $n(0)=n(1)$ and $n_{x}(0)=n_{x}(1)$,

$$
\int_{0}^{1} \frac{n_{x}^{2} n_{x x}}{2 n^{2}} d x=\frac{1}{3} \int_{0}^{1} \frac{\left(n_{x}^{3}\right)_{x}}{2 n^{2}} d x=\frac{1}{3} \int_{0}^{1} \frac{n_{x}^{4}}{n^{3}} d x+\frac{1}{3}\left[\frac{n_{x}^{3}}{2 n^{2}}\right]_{0}^{1}=\frac{1}{3} \int_{0}^{1} \frac{n_{x}^{4}}{n^{3}} d x,
$$


the third integral in (3.15) becomes

$$
\nu \frac{\varepsilon^{2}}{12} \int_{0}^{1}\left(\frac{n_{x x}^{2}}{n}-\frac{n_{x}^{2} n_{x x}}{2 n^{2}}\right) d x=\nu \frac{\varepsilon^{2}}{3} \int_{0}^{1}\left((\sqrt{n})_{x x}^{2}+\frac{n_{x}^{4}}{48 n^{3}}\right) d x .
$$

Multiplying the Poisson equation (3.3) by $n-1$ and integrating by parts yields

$$
\lambda^{2} \int_{0}^{1} V_{x} n_{x} d x=-\int_{0}^{1}(n-1)(n-C(x)) d x \leq-\frac{1}{2} \int_{0}^{1} n^{2} d x+c_{1},
$$

where $c_{1}=\left(1+\|C\|_{L^{2}}^{2}\right) / 2$. Furthermore, it follows from $J=-\nu n_{x}+J_{0}$ that

$$
\int_{0}^{1} \frac{J^{2}}{\tau n} d x=\frac{J_{0}^{2}}{\tau} \int_{0}^{1} \frac{d x}{n}+\frac{4 \nu^{2}}{\tau} \int_{0}^{1}(\sqrt{n})_{x}^{2} d x
$$

since the integral over the mixed term $\int_{0}^{1}(\log n)_{x} d x$ vanishes in view of the boundary conditions $n(0)=n(1)=1$. Therefore, we obtain from (3.15), divided by $\nu$,

$$
\begin{aligned}
& \int_{0}^{1}\left(\frac{1}{n^{3}}\left(J_{x} n-J n_{x}\right)^{2}+\frac{\varepsilon^{2}}{3}(\sqrt{n})_{x x}^{2}+\frac{\varepsilon^{2}}{144} \frac{n_{x}^{4}}{n^{3}}+\frac{n^{2}}{2 \lambda^{2}}+4\left(T+\frac{\nu}{\tau}\right)(\sqrt{n})_{x}^{2}\right) d x \\
\leq & -\frac{J_{0}^{2}}{\tau \nu} \int_{0}^{1} \frac{d x}{n}+\frac{J_{0} U}{\nu}+\frac{c_{1}}{\lambda^{2}} .
\end{aligned}
$$

It remains to estimate the term $J_{0} U / \nu$. For this we employ Young's inequality,

$$
\frac{J_{0} U}{\nu} \leq \frac{J_{0}^{2}}{2 \tau \nu} \int_{0}^{1} \frac{d x}{n}+\frac{\tau U^{2}}{2 \nu} \int_{0}^{1} n d x .
$$

The first term on the right-hand side can be absorbed by the corresponding term in (3.16). We employ again Young's inequality to treat the second term:

$$
\begin{aligned}
\frac{\tau U^{2}}{2 \nu} \int_{0}^{1} n d x & =\frac{\tau U^{2}}{2 \nu}\|\sqrt{n}\|_{L^{2}}^{2} \leq \frac{\tau U^{2}}{2 \nu}\|\sqrt{n}\|_{L^{4}}^{2} \\
& \leq \frac{1}{4 \lambda^{2}}\|\sqrt{n}\|_{L^{4}}^{4}+\frac{\lambda^{2} \tau^{2} U^{4}}{4 \nu^{2}}=\frac{1}{4 \lambda^{2}} \int_{0}^{1} n^{2} d x+\frac{\lambda^{2} \tau^{2} U^{4}}{4 \nu^{2}} .
\end{aligned}
$$

Putting these inequalities into (3.16), we obtain

$$
\begin{aligned}
& \int_{0}^{1}\left(\frac{1}{n^{3}}\left(J_{x} n-J n_{x}\right)^{2}+\frac{\varepsilon^{2}}{3}(\sqrt{n})_{x x}^{2}+\frac{\varepsilon^{2}}{144} \frac{n_{x}^{4}}{n^{3}}+\frac{n^{2}}{4 \lambda^{2}}+4\left(T+\frac{\nu}{\tau}\right)(\sqrt{n})_{x}^{2}\right) d x \\
\leq & -\frac{J_{0}^{2}}{2 \tau \nu} \int_{0}^{1} \frac{d x}{n}+\frac{\lambda^{2} \tau^{2} U^{4}}{4 \nu^{2}}+\frac{c_{1}}{\lambda^{2}} .
\end{aligned}
$$

This shows the lemma.

The following a priori estimates are consequences of the previous lemma.

LEMMA 3.4. Let $(n, J, V) \in H^{4}(0,1) \times H^{3}(0,1) \times H^{2}(0,1)$ be a solution to (3.1)-(3.5) satisfying $n>0$ in $(0,1)$. Then there exists a constant $c>0$ such that

$$
\left|J_{0}\right| \leq c \nu^{-1}, \quad\left|J_{0}\right|\left\|\frac{1}{\sqrt{n}}\right\|_{H^{1}}^{2} \leq c \nu^{-1}
$$


where $J_{0}=J-\nu n_{x}$ and $c$ only depends on $\tau, \lambda, U$, and $\|C\|_{L^{2}}$.

If $U=0,(3.12)$ shows that $J_{0}=0$, and hence the above estimates become trivial. A consequence of the above lemma is the boundedness of the velocity $J_{0} / n$ in $L^{\infty}(0,1)$.

Proof. Lemma 3.3 immediately gives the bound $\|\sqrt{n}\|_{L^{\infty}} \leq c \nu^{-1}$, since $H^{2}(0,1)$ embeds into $L^{\infty}(0,1)$. (Here and in the following, $c>0$ denotes a generic constant not depending on $\nu$.) Thus, since $J_{0}^{2} \int_{0}^{1} d x / n$ is uniformly bounded,

$$
J_{0}^{2} \leq J_{0}^{2} \int_{0}^{1} \frac{d x}{n}\|\sqrt{n}\|_{L^{\infty}}^{2} \leq c\|\sqrt{n}\|_{L^{\infty}}^{2} \leq c \nu^{-2},
$$

which shows the first assertion.

If $U>0$ then, by (3.12), $J_{0}>0$ and

$$
J_{0} \int_{0}^{1} \frac{n_{x}^{2}}{n^{3}} d x=\frac{U}{2 \nu}-\frac{J_{0}}{2 \tau \nu} \int_{0}^{1} \frac{d x}{n} \leq \frac{U}{2 \nu} .
$$

On the other hand, if $U<0$ then $J_{0}<0$ and

$$
-J_{0} \int_{0}^{1} \frac{n_{x}^{2}}{n^{3}} d x=-\frac{U}{2 \nu}+\frac{J_{0}}{2 \tau \nu} \int_{0}^{1} \frac{d x}{n} \leq-\frac{U}{2 \nu} .
$$

This shows that

$$
\left|J_{0}\right| \int_{0}^{1}\left|\left(\frac{1}{\sqrt{n}}\right)_{x}\right|^{2} d x=\frac{\left|J_{0}\right|}{4} \int_{0}^{1} \frac{n_{x}^{2}}{n^{3}} d x \leq \frac{|U|}{8 \nu} .
$$

By Poincaré's inequality, we have

$$
\left|J_{0}\right| \int_{0}^{1}\left(\frac{1}{2 n}-1\right) d x \leq\left|J_{0}\right| \int_{0}^{1}\left(\frac{1}{\sqrt{n}}-1\right)^{2} d x \leq c\left|J_{0}\right|\left\|\left(\frac{1}{\sqrt{n}}\right)_{x}\right\|_{L^{2}}^{2} \leq c \nu^{-1},
$$

and hence, using the first assertion,

$$
\frac{\left|J_{0}\right|}{2}\left\|\frac{1}{\sqrt{n}}\right\|_{L^{2}}^{2} \leq c \nu^{-1}+\left|J_{0}\right| \leq c \nu^{-1} .
$$

The second assertion is proved.

Lemma 3.5. Let $(n, J, V) \in H^{4}(0,1) \times H^{3}(0,1) \times H^{2}(0,1)$ be a solution to (3.1)-(3.5) satisfying $n>0$ in $(0,1)$. Then there exists a constant $c>0$ such that for all $\nu<1$,

$$
\|\log n\|_{H^{2}} \leq c \nu^{-1},
$$

and $c>0$ only depends on $T, \varepsilon, \lambda, U$, and $\|C\|_{L^{2}}$.

Proof. By Lemma 3.2, $w=\log n$ solves (3.9). Since $w \in H_{\text {per }}^{2}(0,1)$, where $H_{\text {per }}^{2}(0,1)$ is the space of all periodic $H^{2}$ functions, we can use $w$ as a test function in the weak formulation of (3.9) leading to

$$
\begin{aligned}
& \left(\nu^{2}+\frac{\varepsilon^{2}}{12}\right) \int_{0}^{1} w_{x x}^{2} d x+\left(T+\frac{\nu}{\tau}\right) \int_{0}^{1} w_{x}^{2} d x \\
= & -\lambda^{-2} \int_{0}^{1}\left(\mathrm{e}^{w}-C\right) w d x+J_{0}^{2} \int_{0}^{1} \mathrm{e}^{-2 w} w_{x}^{2} d x \\
& -2 J_{0} \nu \int_{0}^{1} \mathrm{e}^{-w} w_{x x} w_{x} d x-\frac{J_{0}}{\tau} \int_{0}^{1} \mathrm{e}^{-w} w_{x} d x .
\end{aligned}
$$


The last integral on the right-hand side vanishes since $w(0)=w(1)$. The function $x \mapsto-x \mathrm{e}^{x}, x \in \mathbb{R}$, has the maximal value $\mathrm{e}^{-1}$. Hence, the first integral on the righthand side can be estimated as follows, employing Poincaré's and Young's inequalities,

$$
\begin{aligned}
-\lambda^{-2} \int_{0}^{1}\left(\mathrm{e}^{w}-C\right) w d x & \leq \lambda^{-2} \int_{0}^{1}\left(\mathrm{e}^{-1}+C w\right) d x \leq \lambda^{-2}\left(1+\|C\|_{L^{2}}\|w\|_{L^{2}}\right) \\
& \leq c+\frac{T}{2}\left\|w_{x}\right\|_{L^{2}}^{2}
\end{aligned}
$$

where $c>0$ depends on $T$. Next, we consider the second integral:

$$
\begin{aligned}
J_{0}^{2} \int_{0}^{1} \mathrm{e}^{-2 w} w_{x}^{2} d x & \leq\left\|J_{0} \mathrm{e}^{-w}\right\|_{L^{\infty}}\left|J_{0}\right| \int_{0}^{1} \mathrm{e}^{-w} w_{x}^{2} d x \\
& =\left\|\frac{J_{0}}{n}\right\|_{L^{\infty}} \cdot\left|J_{0}\right| \int_{0}^{1} \frac{n_{x}^{2}}{n^{3}} d x \leq c \nu^{-1} \cdot c \nu^{-1}=c \nu^{-2}
\end{aligned}
$$

using Lemma 3.4. This inequality allows to treat the remaining third integral:

$$
\begin{aligned}
-2 J_{0} \nu \int_{0}^{1} \mathrm{e}^{-w} w_{x x} w_{x} d x & \leq \frac{\varepsilon^{2}}{24} \int_{0}^{1} w_{x x}^{2} d x+\frac{24 \nu^{2} J_{0}^{2}}{\varepsilon^{2}} \int_{0}^{1} \mathrm{e}^{-2 w} w_{x}^{2} d x \\
& \leq \frac{\varepsilon^{2}}{24} \int_{0}^{1} w_{x x}^{2} d x+c
\end{aligned}
$$

Putting together the above estimates gives, for $\nu<1$,

$$
\left(\nu^{2}+\frac{\varepsilon^{2}}{24}\right) \int_{0}^{1} w_{x x}^{2} d x+\left(\frac{T}{2}+\frac{\nu}{\tau}\right) \int_{0}^{1} w_{x}^{2} d x \leq c \nu^{-2} .
$$

Then the conclusion follows from Poincaré's inequality.

3.3. Existence of solutions to (3.9) and (3.11).

Lemma 3.6. Let $C \in L^{2}(0,1)$. Then there exists a solution $w \in H^{4}(0,1)$ to (3.9) and (3.11).

Proof. The proof is based on Leray-Schauder's fixed-point theorem and the a priori estimate of Lemma 3.5. First, we consider weak solutions. Let $H=H_{0}^{1}(0,1) \cap$ $H_{\text {per }}^{2}(0,1)$. As usual, $w \in H$ is called a weak solution to (3.9) and (3.11) if, for $J_{0} \in \mathbb{R}$ given, for all $\phi \in H$,

$$
\begin{aligned}
& -\left(\nu^{2}+\frac{\varepsilon^{2}}{12}\right) \int_{0}^{1}\left(w_{x x}+\frac{1}{2} w_{x}^{2}\right) \phi_{x x} d x-\left(T+\frac{\nu}{\tau}\right) \int_{0}^{1} w_{x} \phi_{x} d x \\
= & \lambda^{-2} \int_{0}^{1}\left(\mathrm{e}^{w}-C\right) \phi d x-J_{0}^{2} \int_{0}^{1} \mathrm{e}^{-2 w} w_{x} \phi_{x} d x \\
& +2 J_{0} \nu \int_{0}^{1} \mathrm{e}^{-w} w_{x x} \phi_{x} d x+\frac{J_{0}}{\tau} \int_{0}^{1} \mathrm{e}^{-w} \phi_{x} d x .
\end{aligned}
$$

For the definition of the fixed-point operator, let $v \in W^{1,4}(0,1), I_{0} \in \mathbb{R}$, and $\sigma \in[0,1]$ be given. We wish to solve the following linear problem in $H$ :

$$
a(w, \phi)=\sigma F(\phi) \text { for all } \phi \in H
$$


where for all $w, \phi \in H$,

$$
\begin{aligned}
a(w, \phi)= & \left(\nu^{2}+\frac{\varepsilon^{2}}{12}\right) \int_{0}^{1} w_{x x} \phi_{x x} d x+\left(T+\frac{\nu}{\tau}\right) \int_{0}^{1} w_{x} \phi_{x} d x \\
F(\phi)= & -\frac{1}{2}\left(\nu^{2}+\frac{\varepsilon^{2}}{12}\right) \int_{0}^{1} v_{x}^{2} \phi_{x x} d x-\lambda^{-2} \int_{0}^{1}\left(\mathrm{e}^{v}-C\right) d x \\
& +I_{0}^{2} \int_{0}^{1} \mathrm{e}^{-2 v} v_{x} \phi_{x} d x+2 \nu I_{0} \int_{0}^{1}\left(\mathrm{e}^{-v} \phi_{x}\right)_{x} v_{x} d x-\frac{I_{0}}{\tau} \int_{0}^{1} \mathrm{e}^{-v} \phi_{x} d x
\end{aligned}
$$

Notice that the product $v_{x}^{2} \phi_{x x}$ is integrable since $v \in W^{1,4}(0,1)$. The bilinear form $a(\cdot, \cdot)$ is continuous and coercive in $H$ since we can apply Poincaré's inequality. Furthermore, the linear functional $F$ is continuous. Hence, Lax-Milgram's lemma provides the existence of a unique solution $w \in H$ to (3.18). Finally, we define $J_{0}$ as the solution to (see (3.12))

$$
\sigma U=J_{0}\left(\frac{1}{\tau} \int_{0}^{1} \mathrm{e}^{-w} d x+2 \nu \int_{0}^{1} \mathrm{e}^{-w} w_{x}^{2} d x\right) .
$$

Clearly, if $U=0$ then $J_{0}=0$.

Thus we can define the fixed-point operator

$$
S:\left(W^{1,4}(0,1) \times \mathbb{R}\right) \times[0,1] \rightarrow W^{1,4}(0,1) \times \mathbb{R}
$$

by $S\left(v, I_{0}, \sigma\right)=\left(w, J_{0}\right)$. By Poincaré's inequality, $S\left(v, I_{0}, 0\right)=(0,0)$. By standard arguments, $S$ is continuous and, since the range of $S$ is contained in $H^{2}(0,1)$ and $H^{2}(0,1)$ embeds compactly into $W^{1,4}(0,1), S$ is also compact. It remains to show that there is a constant $c>0$ such that $\|w\|_{W^{1,4}}+\left|J_{0}\right| \leq c$ for all fixed points $\left(w, J_{0}\right)$ of $S(\cdot, \cdot, \sigma)$.

Let $\left(w, J_{0}\right) \in H \times \mathbb{R}$ be a fixed point of $S$. Then $w \in H$ satisfies (3.9) in the sense of $H^{-2}(0,1)$. Since $\left(w_{x}^{2}\right)_{x x}, \quad\left(\mathrm{e}^{-w} w_{x x}\right)_{x} \in H^{-1}(0,1)$, this equation shows that $w_{x x x x} \in H^{-1}(0,1)$ and $w_{x x x} \in L^{2}(0,1)$. Employing (3.9) again, we obtain $w_{x x x x} \in$ $L^{2}(0,1)$ and thus $w \in H^{4}(0,1)$, i.e., (3.9) is satisfied pointwise in $(0,1)$. Moreover, $V \in H^{2}(0,1)$ by $(3.10)$. (In fact, $V$ is much more regular, but we do not need this fact.) Thus, $(n, J, V) \in H^{4}(0,1) \times H^{3}(0,1) \times H^{2}(0,1)$ with $n=\mathrm{e}^{w}>0$, and $J=\nu n_{x}+J_{0}$ solves (3.1) (3.5). In particular, Lemmas 3.4 and 3.5 can be applied providing uniform bounds for $\left|J_{0}\right|$ and $w=\log n$ in $H^{2}(0,1)$. More precisely, this settles the case $\sigma=1$; however, it is not difficult to see that a similar estimate also holds for $\sigma<1$. Hence, Leray-Schauder's fixed-point theorem gives a solution to (3.17) and thus to (3.9) and (3.11).

\section{Numerical results}

In this section we discretize and numerically solve the viscous and the inviscid QHD equations using finite differences.

4.1. Numerical discretization. The stationary viscous QHD model is numerically solved in one space dimension. In this situation, the temperature in (2.7) can be replaced by the energy density and a term involving $n(\log n)_{x x}$, which can be summarized with the quantum term in (2.7). This leads to the following set of 
equations in the variables $n, J$, and ne (and the potential $V$ ):

$$
\begin{aligned}
J_{x}= & \nu n_{x x} \\
& \frac{2}{3}\left(\frac{J^{2}}{n}\right)+\frac{2}{3}(n e)_{x}-n V_{x}-\frac{\varepsilon^{2}}{18}\left(n(\log n)_{x x}\right)_{x} \\
= & -\frac{J}{\tau}+\nu J_{x x}-\mu n_{x}, \\
& \frac{5}{3}\left(\frac{J n e}{n}\right)-\left(\frac{J^{3}}{3 n^{2}}\right)_{x}-J V_{x}-\frac{\varepsilon^{2}}{18}\left(J(\log n)_{x x}\right)_{x} \\
= & -\frac{2}{\tau}\left(n e-\frac{3}{2} n\right)+\nu(n e)_{x x}-\mu J_{x}, \\
\lambda^{2} V_{x x}= & n-C(x) .
\end{aligned}
$$

We define a uniform mesh by $x_{i}=i h(i=0, \ldots, N)$, where $h=1 / N$ is the mesh size. In order to discretize the Neumann boundary conditions, we introduce the ghost cells $\left[x_{-1}, x_{0}\right]$ and $\left[x_{N}, x_{N+1}\right]$, where $x_{-1}=-h$ and $x_{N+1}=(n+1) h$.

First we discretize the viscous QHD equations using a central finite-difference scheme. For this, the electron density $n$ and electric potential $V$ are approximated at the grid points $x_{i}$, whereas the current density $J$ and the energy density ne are discretized in the mid-points $x_{i-1 / 2}=\left(x_{i}+x_{i-1}\right) / 2$. We denote by $n_{i}$ and $V_{i}$ the approximations of $n\left(x_{i}\right)$ and $V\left(x_{i}\right)$ and by $J_{i-1 / 2}$ and $n e_{i-1 / 2}$ the approximations of $J\left(x_{i-1 / 2}\right)$, and $n e\left(x_{i-1 / 2}\right)$, respectively. For the sake of completeness, and since we analyze the numerical scheme in the next subsection, we make explicit the discretization. The central finite-difference scheme for (4.1) and (4.4) at $x=x_{i}$ reads as

$$
\begin{aligned}
& 0=\frac{J_{i+1 / 2}-J_{i-1 / 2}}{h}-\frac{\nu}{h^{2}}\left(n_{i+1}-2 n_{i}+n_{i-1}\right), \\
& 0=-\frac{\lambda^{2}}{h^{2}}\left(V_{i+1}-2 V_{i}+V_{i-1}\right)+n_{i}-C_{i},
\end{aligned}
$$

where $C_{i}=C\left(x_{i}\right)$ and $i=1, \ldots, N-1$. The central discretization of $(4.2)$ at $x=x_{i-1 / 2}$ is

$$
\begin{aligned}
0=\frac{1}{6 h} & \left(\frac{\left(J_{i+1 / 2}+J_{i-1 / 2}\right)^{2}}{n_{i}}-\frac{\left(J_{i-1 / 2}+J_{i-3 / 2}\right)^{2}}{n_{i-1}}\right) \\
& +\frac{1}{3 h}\left(n e_{i+1 / 2}-n e_{i-3 / 2}\right)-\frac{n_{i}+n_{i-1}}{2} \frac{V_{i}-V_{i-1}}{h} \\
& -\frac{\varepsilon^{2}}{18 h^{3}}\left(n_{i} \log \frac{n_{i+1} n_{i-1}}{n_{i}^{2}}-n_{i-1} \log \frac{n_{i} n_{i-2}}{n_{i-1}^{2}}\right) \\
& +\frac{J_{i-1 / 2}}{\tau}+\frac{\mu}{h}\left(n_{i}-n_{i-1}\right)-\frac{\nu}{h^{2}}\left(J_{i+1 / 2}-2 J_{i-1 / 2}+J_{i-3 / 2}\right)
\end{aligned}
$$

and the central discretization of (4.3) at $x=x_{i-1 / 2}$ reads as

$$
\begin{aligned}
0= & \frac{5}{12 h}\left(\frac{\left(J_{i+1 / 2}+J_{i-1 / 2}\right)\left(n e_{i+1 / 2}+n e_{i-1 / 2}\right)}{n_{i}}\right. \\
& \left.-\frac{\left(J_{i-1 / 2}+J_{i-3 / 2}\right)\left(n e_{i-1 / 2}+n e_{i-3 / 2}\right)}{n_{i-1}}\right) \\
& -\frac{1}{24 h}\left(\frac{\left(J_{i+1 / 2}+J_{i-1 / 2}\right)^{3}}{n_{i}^{2}}-\frac{\left(J_{i-1 / 2}+J_{i-3 / 2}\right)^{3}}{n_{i-1}^{2}}\right)-J_{i-1 / 2} \frac{V_{i}-V_{i-1}}{h}
\end{aligned}
$$




$$
\begin{aligned}
& -\frac{\varepsilon^{2}}{36 h^{3}}\left(\left(J_{i+1 / 2}+J_{i-1 / 2}\right) \log \frac{n_{i+1} n_{i-1}}{n_{i}^{2}}-\left(J_{i-1 / 2}+J_{i-3 / 2}\right) \log \frac{n_{i} n_{i-2}}{n_{i-1}^{2}}\right) \\
& +\frac{2}{\tau}\left(n e_{i-1 / 2}-\frac{3}{4}\left(n_{i}+n_{i-1}\right)\right)+\frac{\mu}{2 h}\left(J_{i+1 / 2}-J_{i-3 / 2}\right) \\
& -\frac{\nu}{h^{2}}\left(n e_{i+1 / 2}-2 n e_{i-1 / 2}+n e_{i-3 / 2}\right),
\end{aligned}
$$

where $i=1, \ldots, N$.

We impose the following boundary conditions:

$$
\begin{aligned}
n_{0} & =C_{0}, \quad n_{N}=C_{N}, \quad n_{1}=n_{-1}, \quad n_{N-1}=n_{N+1}, \\
J_{1 / 2} & =J_{-1 / 2}, \quad J_{N+1 / 2}=J_{N-1 / 2}, n e_{1 / 2}=n e_{-1 / 2}, n e_{N+1 / 2}=n e_{N-1 / 2}, \\
V_{0} & =0, \quad V_{N}=U .
\end{aligned}
$$

With these ten conditions, the discrete system seems to be overdetermined. However, the choice of the doping profile of the tunnelling diode simulated in section $4.3 \mathrm{im}$ plies that the particle density fulfills approximately the conditions $n=C, J=\nu n_{x}=0$, and $J_{x}=\nu n_{x x}=0$ at the boundary such that the discrete system is practically not overdetermined.

Next, we turn to the discretization of the inviscid QHD model using second upwind finite differences first employed in [14]. The QHD equations are here written in the variables $n, u=J / n$, and $T$ rather than in $n, J$, and ne. Furthermore, the variables $n, T$, and $V$ are approximated at the grid points $x_{i}$, but only $u$ is discretized at the mid-points $x_{i-1 / 2}$. The QHD system can be formulated in the form $(u g)_{x}+$ $f=0$, where $g, f \in \mathbb{R}^{4}$ are appropriate vector-valued functions, namely, writing $g=$ $\left(g^{(1)}, g^{(2)}, g^{(3)}, g^{(4)}\right)$,

$$
\begin{aligned}
& g^{(1)}=n, \quad g^{(2)}=n u, \\
& g^{(3)}=\frac{5}{2} n T+\frac{1}{2} n u^{2}-n V-\frac{\varepsilon^{2}}{12}\left(n(\log n)_{x x}\right)_{x}, \quad g^{(4)}=0 .
\end{aligned}
$$

The advection terms $\left(n g^{(j)}\right)_{x}$ are discretized using second upwind differences. More precisely, $(n u)_{x}$ at $x_{i}$ is discretized by

$$
A_{1}=\frac{1}{h}\left(u_{i+1 / 2} n_{i+1 / 2}^{\text {up }}-u_{i-1 / 2} n_{i-1 / 2}^{\text {up }}\right),
$$

where

$$
n_{i+1 / 2}^{\text {up }}= \begin{cases}n_{i} & \text { if } u_{i+1 / 2}>0, \\ n_{i+1} & \text { if } u_{i+1 / 2}<0 .\end{cases}
$$

The term $\left(n u^{2}\right)_{x}$ is approximated at $x_{i+1 / 2}$ by

$$
A_{2}=\frac{1}{h}\left(u_{i+1}(n u)_{i+1}^{\mathrm{up}}-u_{i}(n u)_{i}^{\mathrm{up}}\right),
$$

where

$$
(n u)_{i+1}^{\mathrm{up}}= \begin{cases}\frac{1}{2}\left(n_{i}+n_{i+1}\right) u_{i+1 / 2} & \text { if } u_{i+1}>0 \\ \frac{1}{2}\left(n_{i+1}+n_{i+2}\right) u_{i+3 / 2} & \text { if } u_{i+1}<0\end{cases}
$$


The remaining term $\left(n g^{(3)}\right)_{x}$ is discretized at $x_{i+1 / 2}$ in a similar way noticing that $g^{(3)}\left(x_{i}\right)$ is approximated by

$$
g_{i}^{(3)}=\frac{5}{2} n_{i} T_{i}+\frac{1}{8} n_{i}\left(u_{i-1 / 2}+u_{i+1 / 2}\right)^{2}-n_{i} V_{i}-\frac{\varepsilon^{2}}{12 h^{2}} n_{i} \log \left(\frac{n_{i+1} n_{i-1}}{n_{i}^{2}}\right) .
$$

Gardner has imposed the following boundary conditions

$$
\begin{aligned}
& n(0)=C(0), \quad n(1)=C(1), \quad n_{x}(0)=n_{x}(1)=0, \\
& T(0)=T(1)=1, \quad V(0)=0, \quad V(1)=U,
\end{aligned}
$$

which are discretized similar as in (4.9)-(4.11).

The above discrete nonlinear system is solved by using Newton's method and the line search method of [10] (Algorithm A6.3.1, p. 325). For a given applied voltage, we employed the continuation method, i.e., with the solution for the applied voltage $U$ as an initial guess, we solve the problem applying the potential $U+\triangle U$ and use this solution again as an initial guess for the next applied voltage. For the computations in section 4.3, we have chosen $\triangle U=1 \mathrm{mV}$ and the final voltage is usually $U=0.5 \mathrm{~V}$. The number of grid points is $N=1000$ such that $h=1 / N=10^{-3}$.

4.2. Numerical viscosity and numerical dispersion. In this section, we analyze the finite-difference schemes of the previous subsection. In particular, we derive the consistency error in order to examine the strength of numerical viscosity or dispersion introduced by the discretization.

We start with the viscous QHD model. Let $(n, J, n e, V)$ be a smooth solution to (4.1)-(4.4). To simplify the notation we set $n_{i}=n\left(x_{i}\right), n_{i+1 / 2}=n\left(x_{i+1 / 2}\right)$ etc. Since we consider in this section only exact solutions, no confusion with the notation of the previous section should arise. By standard Taylor expansion, we find that (4.1) can be expanded as

$$
\begin{aligned}
\left(J_{x}+\nu n_{x x}\right)\left(x_{i}\right)= & \frac{1}{h}\left(J_{i+1 / 2}-J_{i-1 / 2}\right)-\frac{\nu}{h^{2}}\left(n_{i+1}-2 n_{i}+n_{i-1}\right) \\
& +\frac{\nu h^{2}}{24} n_{x x x x}\left(x_{i}\right)+O\left(h^{4}\right) .
\end{aligned}
$$

Typical values of $\varepsilon^{2}, \nu$, and $h$ are (see section 4.3) $\varepsilon^{2}=10^{-2}, \nu=10^{-2}, h=10^{-3}$. Thus our central finite-difference discretization involves the numerical fourth-order diffusion $\left(\nu h^{2} / 24\right) n_{x x x x} \approx 10^{-10} n_{x x x x}$.

The expansions for the terms of (4.2) read as follows:

$$
\begin{aligned}
\left(\frac{J^{2}}{n}\right)_{x}\left(x_{i-1 / 2}\right)= & \frac{1}{4 h}\left(\frac{\left(J_{i+1 / 2}+J_{i-1 / 2}\right)^{2}}{n_{i}}-\frac{\left(J_{i-1 / 2}+J_{i-3 / 2}\right)^{2}}{n_{i-1}}\right) \\
& +\frac{h^{2}}{24}\left(\frac{J^{2}}{n}\right)_{x x x}\left(x_{i-1 / 2}\right)+O\left(h^{3}\right), \\
n_{x}\left(x_{i-1 / 2}\right)= & \frac{1}{h}\left(n_{i}-n_{i-1}\right)-\frac{h^{2}}{24} n_{x x x}\left(x_{i-1 / 2}\right)+O\left(h^{4}\right) \\
& n_{i} \log \left(\frac{n_{i+1} n_{i-1}}{n_{i}^{2}}\right)-n_{i-1} \log \left(\frac{n_{i} n_{i-2}}{n_{i-1}^{2}}\right) \\
& +\frac{\varepsilon^{2} h^{2}}{24} n_{x x x}\left(x_{i-1 / 2}\right)+O\left(h^{3}\right), \\
\nu J_{x x}\left(x_{i-1 / 2}\right)= & \frac{\nu}{h^{2}}\left(J_{i+1 / 2}-2 J_{i-1 / 2}+J_{i-3 / 2}\right)-\frac{\nu^{2} h^{2}}{12} n_{x x x x x}\left(x_{i-1 / 2}\right)+O\left(h^{4}\right) .
\end{aligned}
$$


Thus we obtain a numerical dispersion at most of order $\left(h^{2} / 24\right) n_{x x x} \approx 10^{-7} n_{x x x}$ which is much smaller than the physical dispersion being of order $\varepsilon^{2} n_{x x x} \approx 10^{-4} n_{x x x}$.

In a similar way, one can show that the numerical viscosity and diffusion in the central discretization of (4.3) is of order $h^{2}=10^{-6}$ and therefore much smaller than $\varepsilon^{2}$ and $\nu$. Therefore, we expect that the numerical error of the central scheme is rather small. This expectation will be verified numerically in section 4.3.

Next, we turn to the second upwind discretization of the QHD model. The main error comes from the discretization of the advection terms which are only of order one. Setting $u_{j}^{+}=\max \left\{u\left(x_{j}\right), 0\right\}$ and $u_{j}^{-}=-\min \left\{u\left(x_{j}\right), 0\right\}$, we can write the upwind discretization $A_{1}$ of $(n u)_{x}\left(x_{i}\right)$ (see (4.12)) as

$$
\begin{aligned}
A_{1}= & \frac{1}{h}\left(\left(u_{i+1 / 2}^{+} n_{i}-u_{i+1 / 2}^{-} n_{i+1}\right)-\left(u_{i-1 / 2}^{+} n_{i-1}-u_{i-1 / 2}^{-} n_{i}\right)\right) \\
= & \frac{1}{h}\left(-u_{i+1 / 2}^{-} n_{i+1}+\left(u_{i+1 / 2}^{+}+u_{i-1 / 2}^{-}\right) n_{i}-u_{i-1 / 2}^{+} n_{i-1}\right) \\
= & \frac{1}{h}\left(u_{i+1 / 2} n_{i+1 / 2}-u_{i-1 / 2} n_{i-1 / 2}\right) \\
& -\frac{1}{h} u_{i+1 / 2}^{+}\left(n_{i+1 / 2}-n_{i}\right)-\frac{1}{h} u_{i+1 / 2}^{-}\left(n_{i+1}-n_{i+1 / 2}\right) \\
& +\frac{1}{h} u_{i-1 / 2}^{+}\left(n_{i-1 / 2}-n_{i-1}\right)+\frac{1}{h} u_{i-1 / 2}^{-}\left(n_{i}-n_{i-1 / 2}\right) .
\end{aligned}
$$

The central discretization of $n_{x x}$ gives

$$
n_{i+1 / 2}-n_{i}=\frac{1}{2}\left(n_{i+1}-n_{i}\right)+\frac{h^{2}}{8} n_{x x}\left(x_{i+1 / 2}\right)+O\left(h^{4}\right),
$$

and hence, we obtain

$$
\begin{aligned}
A_{1}= & \frac{1}{h}\left(u_{i+1 / 2} n_{i+1 / 2}-u_{i-1 / 2} n_{i-1 / 2}\right) \\
& -\frac{1}{2 h}\left(\left|u_{i+1 / 2}\right|\left(n_{i+1}-n_{i}\right)-\left|u_{i-1 / 2}\right|\left(n_{i}-n_{i-1}\right)\right) \\
& -\frac{h}{8}\left(u_{i+1 / 2} n_{x x}\left(x_{i+1 / 2}\right)-u_{i-1 / 2} n_{x x}\left(x_{i-1 / 2}\right)\right)+O\left(h^{3}\right) .
\end{aligned}
$$

A Taylor expansion shows that this expression can be written as

$$
A_{1}=(u n)_{x}\left(x_{i}\right)-\frac{h}{2}\left(|u| n_{x}\right)_{x}\left(x_{i}\right)+O\left(h^{2}\right) .
$$

The upwind discretization $A_{2}$ of $\left(n u^{2}\right)_{x}\left(x_{i+1 / 2}\right)$ (see (4.13)) can be written as

$$
\begin{aligned}
A_{2} & =\frac{1}{h}\left(n_{i+1} u_{i+1}^{2}-n_{i} u_{i}^{2}\right)+\left(u_{i+1}\left((n u)_{i+1}^{\mathrm{up}}-n_{i+1} u_{i+1}\right)-u_{i}\left((n u)_{i}^{\mathrm{up}}-n_{i} u_{i}\right)\right) \\
& =\frac{1}{h}\left(n_{i+1} u_{i+1}^{2}-n_{i} u_{i}^{2}\right)-\frac{B}{h},
\end{aligned}
$$

where

$$
\begin{aligned}
B= & u_{i+1}^{+}\left(n_{i+1} u_{i+1}-\frac{1}{2}\left(n_{i+1}+n_{i}\right) u_{i+1 / 2}\right) \\
& -u_{i+1}^{-}\left(n_{i+1} u_{i+1}-\frac{1}{2}\left(n_{i+2}+n_{i+1}\right) u_{i+3 / 2}\right) \\
& -u_{i}^{+}\left(n_{i} u_{i}-\frac{1}{2}\left(n_{i}+n_{i-1}\right) u_{i-1 / 2}\right)+u_{i}^{-}\left(n_{i} u_{i}-\frac{1}{2}\left(n_{i+1}+n_{i}\right) u_{i+1 / 2}\right) .
\end{aligned}
$$


Employing the approximation $u_{i}=\left(u_{i+1 / 2}+u_{i-1 / 2}\right) / 2+u^{\prime \prime}\left(x_{i}\right) h^{2} / 4+O\left(h^{3}\right)$ and the relation $u^{ \pm}=(|u| \pm u) / 2$, we obtain after some tedious computations

$$
\begin{aligned}
B= & \frac{1}{4}\left(\left|u_{i+1}\right|\left(\left(n_{i+1}+n_{i+2}\right) u_{i+3 / 2}-\left(n_{i+1}+n_{i}\right) u_{i+1 / 2}\right)\right. \\
& \left.-\left|u_{i}\right|\left(\left(n_{i+1}+n_{i}\right) u_{i+1 / 2}-\left(n_{i}+n_{i-1}\right) u_{i-1 / 2}\right)\right) \\
& +\frac{1}{4}\left(u_{i+1}\left(\left(n_{i+1}-n_{i}\right) u_{i+1 / 2}-\left(n_{i+2}-n_{i+1}\right) u_{i+3 / 2}\right)\right. \\
& \left.-u_{i}\left(\left(n_{i}-n_{i-1}\right) u_{i-1 / 2}-\left(n_{i+1}-n_{i}\right) u_{i+1 / 2}\right)\right)+O\left(h^{3}\right) .
\end{aligned}
$$

This term is a central finite-difference approximation of

$$
h^{2}\left(|u|(n u)_{x}\right)_{x}+h^{3}\left(u\left(u n_{x}\right)_{x}\right)_{x} \quad \text { at } x=x_{i+1 / 2}
$$

such that

$$
A_{2}=\left(n u^{2}\right)_{x}\left(x_{i+1 / 2}\right)+h\left(|u|(n u)_{x}\right)_{x}\left(x_{i+1 / 2}\right)+O\left(h^{2}\right) .
$$

The above calculations show that the upwind scheme introduces diffusion terms for the variables $n$ and $n u$ (with diffusion coefficient $|u|$ ) being of the order $h=10^{-3}$. Since the scaled velocity $|u|$ has numerically values of the order $10^{3}$ (or larger), the numerical diffusion term is of the order $O(1)$ which is much larger than the physical diffusion being of the order $\nu=10^{-2}$.

4.3. Numerical simulations of a resonant tunneling diode. The numerical scheme of section 4.1 is used to simulate a simple one-dimensional resonant tunneling diode. We choose the same geometry and data as in [14]. The tunneling diode consists of highly doped GaAs regions near the contacts and a lightly doped middle region of $25 \mathrm{~nm}$ length (see Figure 4.1). The middle region contains a quantum well of $5 \mathrm{~nm}$ length sandwiched between two $5 \mathrm{~nm}$ AlGaAs barriers. The double barrier heterostructure is placed between two $10 \mathrm{~nm}$ GaAs spacer layers with a doping of $5 \cdot 10^{15} \mathrm{~cm}^{-3}$. These spacers are enclosed by two layers of $50 \mathrm{~nm}$ length and with doping $10^{18} \mathrm{~cm}^{-3}$. The total length is thus $125 \mathrm{~nm}$. The double barrier height is $0.209 \mathrm{eV}$. The physical effect of the barriers is a shift in the quasi-Fermi potential level, which is modeled by an additional step function $V_{\text {ext }}$ added to the electric potential. The physical constants are summarized in Table 4.1.

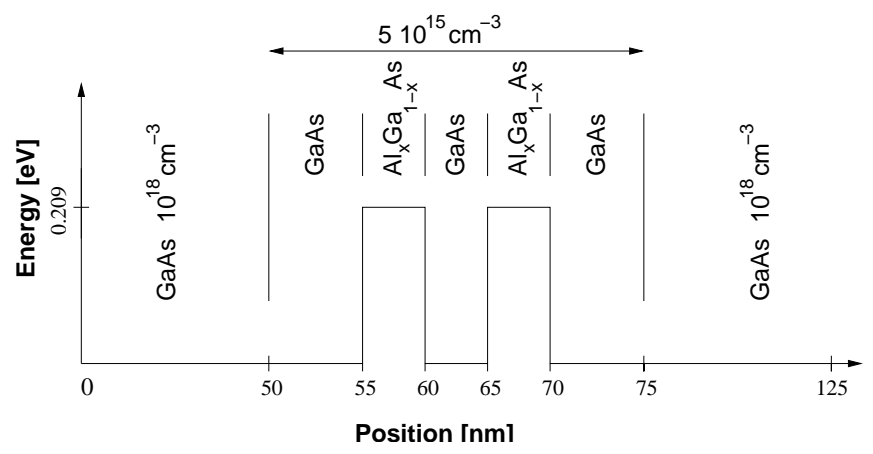

FIG. 4.1. Geometry of the resonant tunneling diode with Al mole fraction $x=0.3$. 


\begin{tabular}{|c|c|c|}
\hline Parameter & Physical meaning & Numerical value \\
\hline$q$ & elementary charge & $1.602 \cdot 10^{-19} \mathrm{As}$ \\
$m_{\mathrm{eff}}$ & effective electron mass & $0.067 \cdot 10^{-31} \mathrm{~kg}$ \\
$k_{B}$ & Boltzmann constant & $1.380 \cdot 10^{-23} \mathrm{~J} / \mathrm{K}$ \\
$\hbar$ & reduced Planck constant & $1.055 \cdot 10^{-34} \mathrm{Js}$ \\
$\varepsilon_{S}$ & semiconductor permitivity & $1.012 \cdot 10^{-10} \mathrm{As} / \mathrm{Vm}$ \\
$T_{0}$ & lattice temperature & $77 \mathrm{~K}$ \\
$\tau_{0}$ & momentum relaxation time & $0.9 \cdot 10^{-12} \mathrm{~s}$ \\
$\nu$ & viscosity & $1.589 \cdot 10^{-5} \mathrm{~m}^{2} / \mathrm{s}^{2}$ \\
\hline
\end{tabular}

TABLE 4.1. Physical parameters for GaAs.
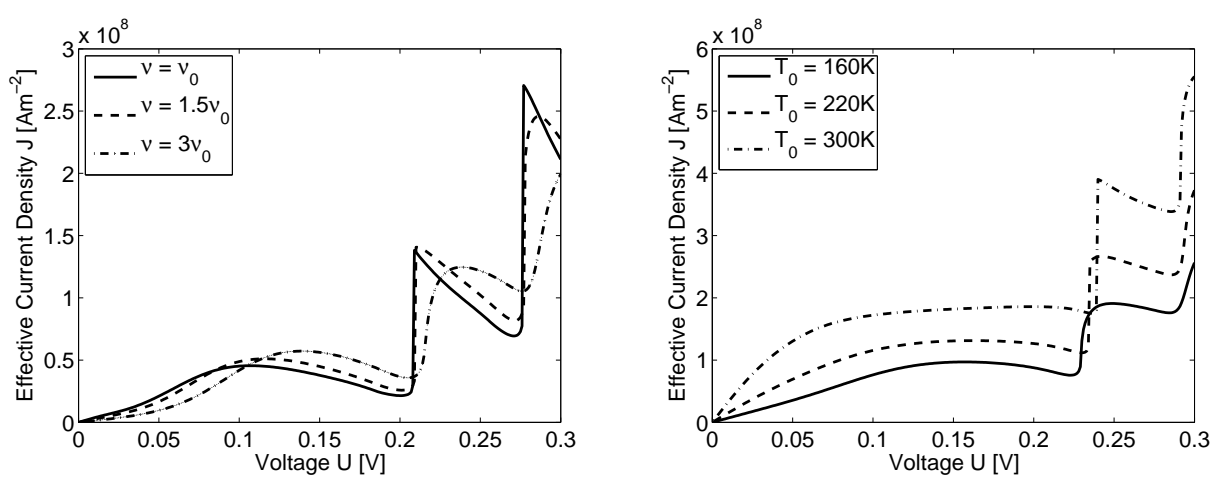

FIG. 4.2. Current-voltage characteristics of a tunneling diode for various values of the viscosity with $\nu_{0}=1.589 \cdot 10^{-5} \mathrm{~m}^{2} / \mathrm{s}^{2}$ (left) and various values of the lattice temperature (right), computed from the isothermal model.

Gardner [14] has added the heat flux term $k_{B} \sigma\left(n T_{x}\right)_{x}$ to the right-hand side of (2.4), where $\sigma=\kappa \tau_{0} k_{B} T_{0} / m$ and $\kappa=0.2$ is the thermal conductivity, for numerical stability. This term is not needed in the numerical solution of the viscous QHD equations, but we used it in the solution of the QHD model. We notice that the values for the momentum relaxation and energy relaxation times are different in the QHD model. We use the same values as in [14].

First, we present numerical results for the isothermal model (4.1), (4.2), (4.4) with constant lattice temperature $T_{0}=77 \mathrm{~K}$ in order to test our numerical algorithm. The stationary numerical solution was already calculated in [28] (as the limit of the transient solution for "large" times) such that our results can be compared to those in [28]. Current-voltage characteristics for the resonant tunneling diode described above are displayed in Figures 4.2 and 4.3. We observe several regions of negative differential resistance (NDR) characterizing the tunneling diode. The curve becomes "smoother" for larger values of the viscosity constant which is expected physically. However, there is a jump of the current density to a larger value after each local minimum; this jump seems to be not physical since in experiments [30], as well as in numerical simulations using the Schrödinger equation (see, e.g., [11]), sharp gradients are observed just after the current peaks. A possible explanation, already given in [28], is that the energy equation needs to be taken into account which will be done below. In fact, we expect that the electrons cool down dramatically as they penetrate 
the potential barriers such that the isothermal model is not accurate enough.

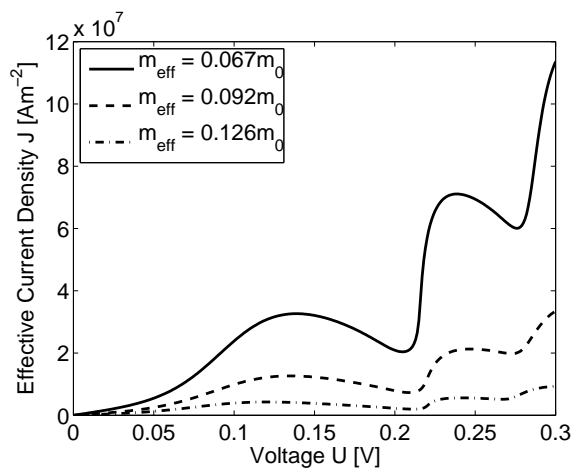

FIG. 4.3. Current-voltage characteristics of a tunneling diode for various values of the effective mass computed from the isothermal model.

It is interesting to observe that there are NDR regions also at room temperature; this is not the case of the quantum drift-diffusion model [27]. In Figure 4.3 the currentvoltage curves for various values of the effective mass constant $\alpha$ with $m_{\text {eff }}=\alpha \cdot m_{0}$ are shown. The peak-to-valley ratios are given in Table 4.2. Interestingly, the peakto-valley ratios are not monotoneous with the effective mass like in the quantum drift-diffusion model [27].

\begin{tabular}{|c|c|c|}
\hline Effective mass & First ratio & Second ratio \\
\hline $0.067 \mathrm{~m}_{0}$ & 1.750 & 1.180 \\
$0.092 \mathrm{~m}_{0}$ & 1.707 & 1.086 \\
$0.126 \mathrm{~m}_{0}$ & 2.205 & 1.108 \\
\hline
\end{tabular}

TABle 4.2. Peak-to-valley ratios from Figure 4.3 for different effective masses.
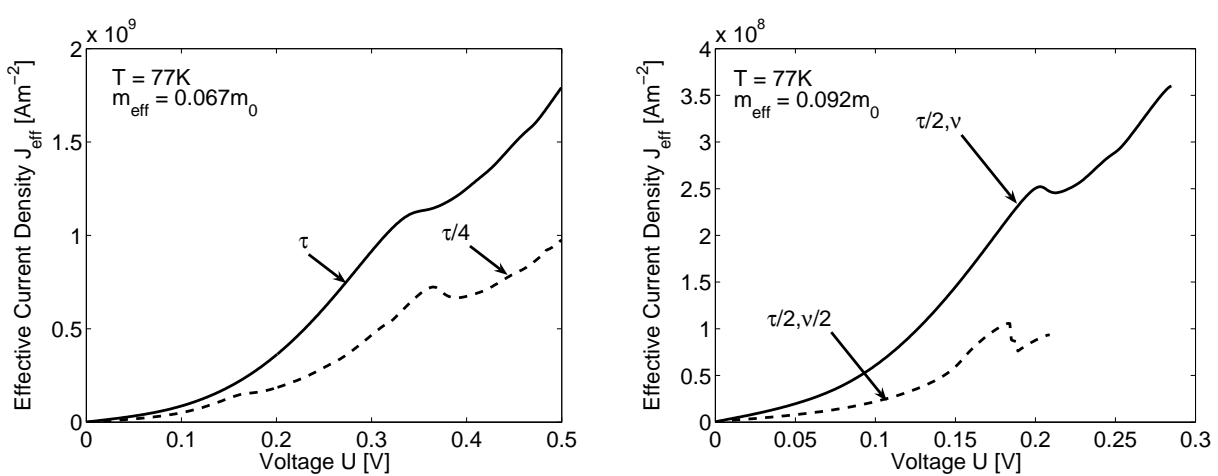

FIG. 4.4. Current-voltage characteristics of a tunneling diode computed from the non-isothermal model with relaxation time $\tau=0.9$ s and viscosity $\nu=1.589 \cdot 10^{-5} \mathrm{~m}^{2} / \mathrm{s}^{2}$.

Next, we turn to the numerical results obtained from the non-isothermal model (4.1)-(4.4). In Figure 4.4 the current-voltage characteristics are shown with lattice 


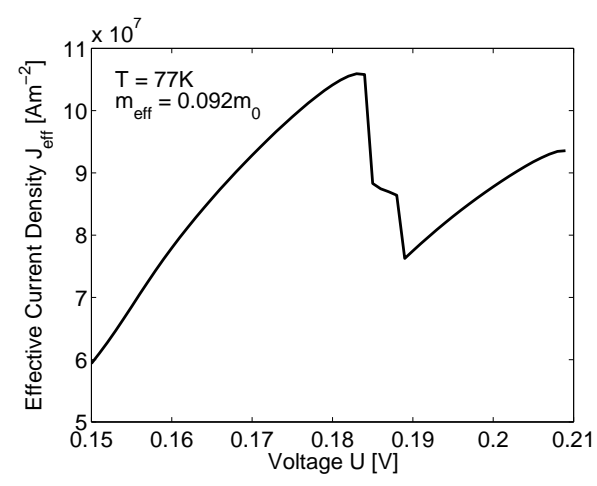

FIG. 4.5. Zoom of Figure 4.4.
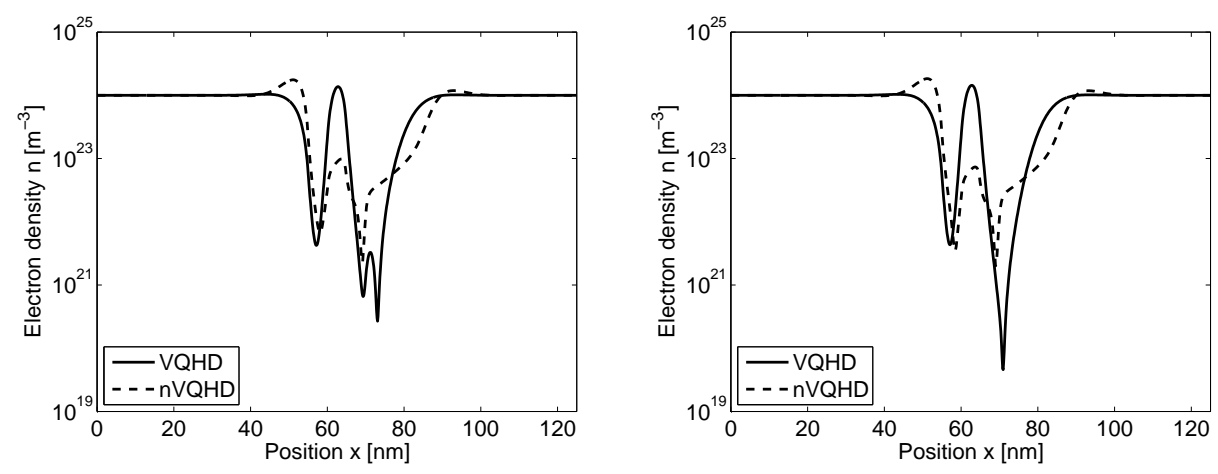

FIG. 4.6. Electron density versus position with relaxation time $\tau / 4=0.225 \mathrm{ps}$ at the peak (left) and valley (right) current.

temperature $T_{0}=77 \mathrm{~K}$. In order to obtain NDR effects, we need to choose a smaller relaxation time than that taken in the isothermal model. The peak-to-valley ratio is too small compared to experiments which may be due to the viscosity. Again, for larger viscosity constants, the current-voltage curves become "smoother". Numerical difficulties (supported by the analytical estimates of section 3) do not allow to perform numerically the inviscid limit $\nu \rightarrow 0$. Interestingly, the current-voltage curve shows a plateau-like behavior (see the zoom in Figure 4.5) which can be also observed experimentally $[3,29]$.

In Figure 4.6, the electron densities for the isothermal and non-isothermal model at the peak and valley current values are displayed. For the non-isothermal model, the data corresponds to the dashed curve in Figure 4.4 (left). The electron density shows a charge enhancement in the quantum well which is more pronounced in the isothermal model. At the center of the right barrier, the electron density dramatically decreases. At the peak current (left figure), the density from the isothermal model develops a "wiggle". This phenomenum is not a numerical effect since it has been observed in various numerical simulations $[28,33]$. This "wiggle" disappears at the valley current where the density becomes very small (right figure). The electron density from the non-isothermal model is "smoother" and its minimum is larger than in the isothermal 

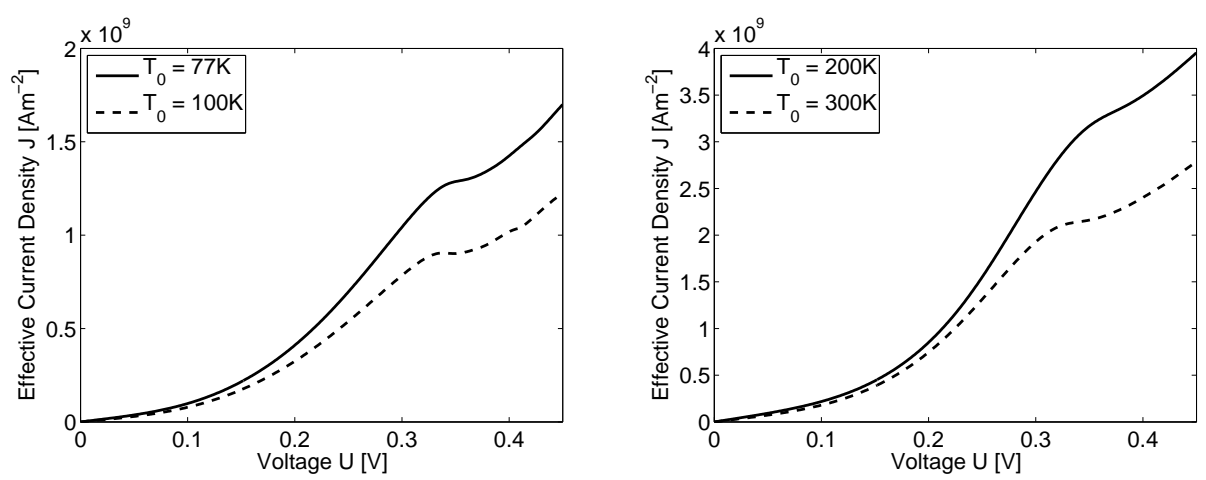

FIG. 4.7. Current-voltage characteristics of a tunneling diode computed from the non-isothermal model for various values of the lattice temperature (left: $\nu=1.589 \cdot 10^{-5} \mathrm{~m}^{2} / \mathrm{s}^{2}$; right: $\nu=5 \cdot 1.589$. $\left.10^{-5} \mathrm{~m}^{2} / \mathrm{s}^{2}\right)$.

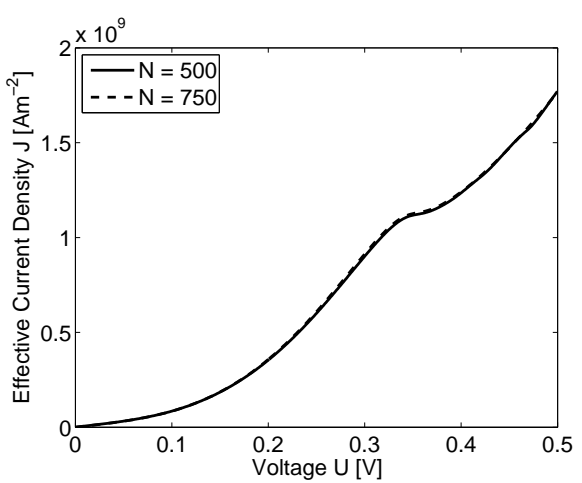

FIG. 4.8. Influence of the number of discretization points on the current-voltage characteristics computed from the non-isothermal viscous model.

model, which stabilizes the numerical scheme.

Next, we investigate the influence of the lattice temperature (see Figure 4.7). In order to compute the solution for $T_{0}=200 \mathrm{~K}$ and $T_{0}=300 \mathrm{~K}$ we needed a larger viscosity constant, namely $5 \nu$. We see that there is no NDR region, even not for small lattice temperature. It seems that the viscosity dominates the quantum effects.

Finally, we notice that the number of discretization points influences the solution behavior only slightly (Figure 4.8) which is to be expected since the central scheme is of second order.

Acknowledgements. The authors acknowledge partial support from the Deutsche Forschungsgemeinschaft (DFG), grants JU359/3 (Gerhard-Hess Award) and JU359/5 (Priority Program "Multi-Scale Problems"), the Wissenschaftkolleg "Differential Equations" financed by the FWF in Austria, and the DAAD-Vigoni Program. This research is part of the ESF program "Global and geometrical aspects of nonlinear partial differential equations (GLOBAL)". 


\section{REFERENCES}

[1] A. Arnold and A. Jüngel, Multi-scale modeling of quantum semiconductor devices, A. Mielke (ed.), Analysis, Modeling and Simulation of Multiscale Problems, Springer, Berlin, 331-363, 2006.

[2] A. Arnold, J. Lopez, P. Markowich and J. Soler, An analysis of quantum Fokker-Planck models: a Wigner function approach, Rev. Mat. Iberoam., 20, 771-814, 2004.

[3] R. C. Bowen, G. Klimeck, R. Lake, W. Frensley and T. Moise, Quantitative simulation of resonant tunneling diode, J. Appl. Phys., 81, 3207-3213, 1997.

[4] D. Bresch, B. Desjardins and C. Lin, On some compressible fluid models: Korteweg, lubrication and shallow water systems, Commun. Part. Diff. Eqs., 28, 1009-1037, 2003.

[5] A. Caldeira and A. Leggett, Path integral approach to quantum Brownian motion, Phys. A, 121A, 587-616, 1983.

[6] F. Castella, L. Erdös, F. Frommlet and P. Markowich, Fokker-Planck equations as scaling limits of reversible quantum systems, J. Stat. Phys., 100, 543-601, 2000.

[7] Z. Chen, A finite element method for the quantum hydrodynamic model for semiconductor devices, Comput. Math. Appl., 31, 17-26, 1996.

[8] Z. Chen, B. Cockburn, C. Gardner and J. Jerome, Quantum hydrodynamic simulation of hysteresis in the resonant tunneling diode, J. Comput. Phys., 117, 274-280, 1995.

[9] P. Degond and C. Ringhofer, Quantum moment hydrodynamics and the entropy principle, J. Stat. Phys., 112, 587-628, 2003.

[10] J. Dennis and R. Schnabel, Numerical Methods for Unconstrained Optimization and Nonlinear Equations, SIAM, Philadelphia, 1996.

[11] A. El Ayyadi and A. Jüngel, Semiconductor simulations using a coupled quantum drift-diffusion Schrödinger-Poisson model, SIAM J. Appl. Math., 66, 554-572, 2005.

[12] D. Ferry and J.-R. Zhou, Form of the quantum potential for use in hydrodynamic equations for semiconductor device modeling, Phys. Rev. B, 48, 7944-7950, 1993.

[13] I. Gamba and A. Jüngel, Positive solutions of singular equations of second and third order for quantum fluids, Archive Rat. Mech. Anal., 156, 183-203, 2001.

[14] C. L. Gardner, The quantum hydrodynamic model for semiconductor devices, SIAM J. Appl. Math., 54, 409-427, 1994.

[15] C. L. Gardner and C. Ringhofer, Smooth quantum potential for the hydrodynamic model, Phys. Rev. E, 53, 157-167, 1996.

[16] C. L. Gardner, G. Klimeck and C. Ringhofer, Smooth quantum hydrodynamic model vs. NEMO simulation of resonant tunneling diodes, J. Comput. Electr., 3, 95-102, 2004.

[17] C. L. Gardner, Private communication, 2007.

[18] I. Gasser, P. A. Markowich, D. Schmidt and A. Unterreiter, Macroscopic theory of charged quantum fluids, in P. Marcati (ed.), Mathematical Problems in Semiconductor Physics, Pitman, Rome, Italy, 340, 42-75, 1995.

[19] I. Gasser and P. A. Markowich, Quantum hydrodynamics, Wigner transforms and the classical limit, Asympt. Anal., 14, 97-116, 1997.

[20] M. P. Gualdani and A. Jüngel, Analysis of the viscous quantum hydrodynamic equations for semiconductors, Europ. J. Appl. Math., 15, 577-595, 2004.

[21] M. P. Gualdani, A. Jüngel and G. Toscani, Exponential decay in time of solutions of the viscous quantum hydrodynamic equations, Appl. Math. Lett., 16, 1273-1278, 2003.

[22] M. T. Gyi and A. Jüngel, A quantum regularization of the one-dimensional hydrodynamic model for semiconductors, Adv. Diff. Eqs., 5, 773-800, 2000.

[23] A. Jüngel, A steady-state quantum Euler-Poisson system for semiconductors, Commun. Math. Phys., 194, 463-479, 1998.

[24] A. Jüngel and H.-L. Li, Quantum Euler-Poisson system: global existence and exponential decay, Quart. Appl. Math., 62, 569-600, 2004.

[25] A. Jüngel, M. C. Mariani and D. Rial, Local existence of solutions to the transient quantum hydrodynamic equations, Math. Models Meth. Appl. Sci., 12, 485-495, 2002.

[26] A. Jüngel, D. Matthes and P. Milišić, Derivation of new quantum hydrodynamic equations using entropy minimization, SIAM J. Appl. Math., 67, 46-68, 2006.

[27] A. Jüngel and J.-P. Milišić, Macroscopic quantum models with and without collisions, Proceedings of the Sixth International Workshop on Mathematical Aspects of Fluid and Plasma Dynamics, Kyoto, Japan., Bulletin Inst. Math., Academia Sinica, to appear, 2007.

[28] A. Jüngel and S. Tang, Numerical approximation of the viscous quantum hydrodynamic model for semiconductors, Appl. Numer. Math., 56, 899-915, 2006.

[29] G. Klimeck, Quantum and semi-classical transport in NEMO 1-D, J. Comput. Electr., 2, 177182,2003 
[30] N. Kluksdahl, A. Kriman, D. Ferry and C. Ringhofer, Self-consistent study of the resonanttunneling diode, Phys. Rev. B, 39, 7720-7735, 1989.

[31] H.-L. Li and P. Marcati, Existence and asymptotic behavior of multi-dimensional quantum hydrodynamic model for semiconductors, Commun. Math. Phys., 245, 215-247, 2004.

[32] E. Madelung, Quantentheorie in hydrodynamischer Form, Z. Physik, 40, 322-326, 1927.

[33] P. Pietra and C. Pohl, Weak limits of the quantum hydrodynamic model, Proceedings of the International Workshop on Quantum Kinetic Theory, Breckenridge, Colorado, USA, Special Issue of VLSI Design, 9, 427-434, 1999.

[34] P. Shukla and L. Stenflo, Stimulated scattering instabilities of electronmagnetic waves in an ultracold quantum plasma, Phys. Plasmas, 13, 044505-044508, 2006.

[35] E. Wigner, On the quantum correction for thermodynamic equilibrium, Phys. Rev., 40, 749-759, 1932. 